\title{
Diatom assemblages of non-living substrates in petrifying Cratoneurion springs from lower Belgium
}

\author{
Luc Denys \& Patrik Oosterlynck
}

Research Institute for Nature and Forest, Kliniekstraat 25, B-1070 Brussels

e-mail: luc.denys@inbo.be

\begin{abstract}
Diatom assemblages were examined from non-living substrates in thirteen springs supporting the Natura 2000 habitat type 'Petryfing springs with tufa formation' in the southern part of Flanders. More than 300 taxa were recorded, including some which appear to be particularly associated with this kind of environment. Even though a number of characteristic taxa and diatoms characterizing fast-flowing springs of more elevated terrain remained absent, local species richness was higher than reported from carbonate springs elsewhere in Europe. Relative abundance of 'Red List' taxa was, however, substantially lower. Taxon richness was not related to measured nutrients but increased with diatom-inferred trophic status. Assemblages consisted mainly of motile, small-celled species of Achnanthidium, Amphora, Caloneis, Planothidium and Stauroneis. Hygropetric rheocrenes presented a somewhat more distinct assemblage than helocrene and rheohelocrene settings, but overall, compositional metrics were similar among spring types. Assemblages were structured mainly along gradients of $\mathrm{pH}$, major ions (in particular magnesium and potassium) and nitrate relating to calcite precipitation, aquifer and nitrogen supply. Besides substantiating broad similarities among Cratoneurion springs, the results provide arguments for a regionalized approach towards assessment of their diversity, community structure and conservation status.
\end{abstract}

Key words: habitats directive, tufa limestone, diversity, carbonate, nitrate, trophic status, regionalization, phytobenthos, crenobiota, Atlantic region

\section{INTRODUCTION}

Worldwide, spring diatoms have drawn considerable attention. This is mainly because the physical and hydrochemical characteristics of these comparatively isolated habitats render their assemblage composition distinct from more downstream parts of the drainage system and because diatoms are prominent primary producers in springs, contributing to conspicuous biogeochemical processes such as limestone formation. Also, because of their upstream location and often considerable hydrological age of the surfacing groundwater, springs may constitute rare havens for pollution-sensitive biota in regions where human impacts on surface water quality are pervasive, whilst environmental heterogeneity and ecoclinal structure warrant higher diversity (GERECKE et al. 2011; CANTONATI et al. 2012a).

Hydrogeological conditions for spring formation occur most frequently in (sub-)mountainous regions with surfacing hard bedrock and a series of recent diatom studies of carbonate springs in Europe have focussed on such areas (e.g., ReICHARDT 1994, 1995,
2004; Cantonati 1998; PoulíčKová et al. 2003; Werum \& LANGe-Bertalot 2004; FránKová et al. 2009; TorRISi \& Dell'Uomo 2009; Gesierich \& Kofler 2010; WoJTAL 2013). Springs also occur in less elevated sloping terrain, wherever groundwater is forced to the surface by less permeable strata, but unlike mid- and highaltitude regions, data on their diatom biodiversity are more restricted and often limited to more mineralpoor conditions. Although a number of previous studies considered diatoms from calcareous low-altitude springs, most concerned regions that are biogeographically distinct from Atlantic NW-Europe and sites at the foot of mountain ranges where the regional species pool is contiguous with headwaters at higher altitude (e.g. Aboal et al. 1998; Angeli et al. 2010; Delgado et al. 2013). In addition, older surveys used a less detailed taxonomy than now customary, making comparisons less straightforward.

The most southern part of Flanders, the northern region of Belgium, where Quaternary loess or Tertiary sands superpose Tertiary aquitards at levels below $200 \mathrm{~m}$ above sea level, is not associated with orogenic terrain. Due to higher carbonate levels in the groundwater and its degassing at emergence, springs in this 
area may present calcite precipitation as well as moss assemblages that allow them to be classified as the Natura 2000 habitat type 7220 'Petrifying springs with tufa formation (Cratoneurion)' (EUROPEAN COMMISSION 2007). Even though this habitat type remains less welldeveloped than its counterparts in more mountainous areas, such springs require particular protection, monitoring and reporting according to the European Habitats Directive. To resolve a general lack of information on the occurrence, abiotic environment and biotic characterisation of petrifying springs in Atlantic Flanders, OOSTERlynck \& De BIE (in press) recently presented a first region-wide survey, focusing primarily on hydrochemical aspects and bryophyte vegetation. This work also provided the opportunity for an initial, albeit more partial exploration of the diatom assemblages associated with the Cratoneurion in lower Belgium, aiming to elucidate its environmental and biological position better within the wider range of carbonate springs in western Europe and, finally, contribute to the assessment of the ecological quality of this habitat type. So far, only Symoens $(1957,960)$ and Iserentant (1988) contributed more than cursory data on the diatom flora of Belgian carbonate springs, neither one considering the Flemish region.

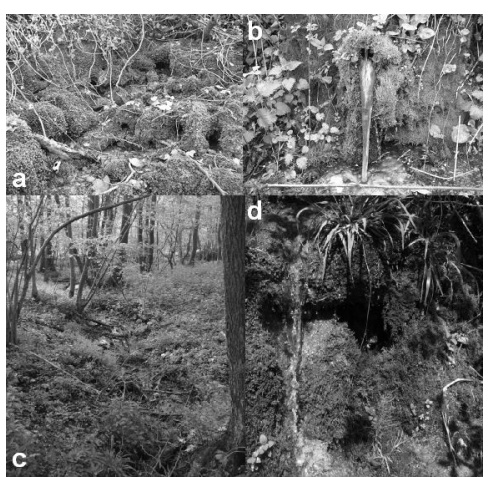

Fig. 1. Examples of Crateuneurion springs in Flanders: (a) Krindaal, rheohelocrene, (KRIND); (b) Boterhoek, tapped source of rheocrene (site BOTER); (c) Mabroek, rheocrene (MABRO); (d) Kwinten, hygropetric rheocrene (KWINT).

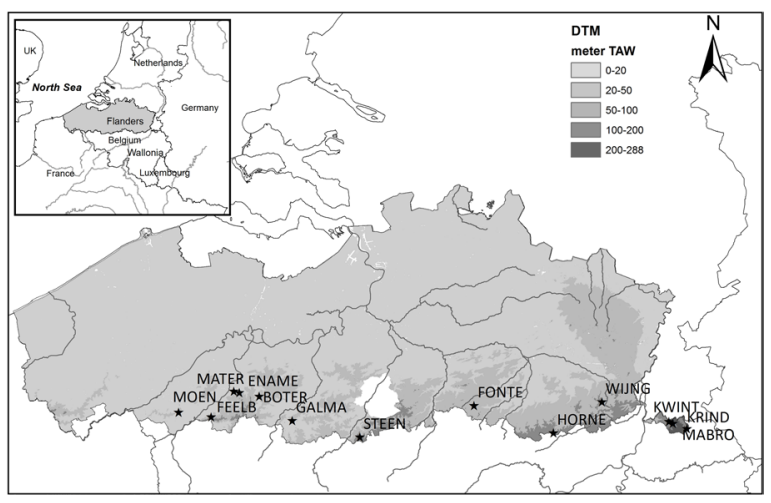

Fig. 2. Topographic map of Flanders with sample locations (TAW: mean sea-level at ebb for Oostende). Insert shows the situation in north-western Europe.

\section{SPRING CHARACTERISTICS}

Spring-associated limestone (SAL) systems (SANDERS et al. 2010) with Cratoneurion in Flanders vary from less welldefined areas with \pm diffuse seepage and limited carbonate deposition on plant debris and stones in discharging seeps (hillslope springs or rheohelocrenes), to distinct sources with encrustations or concretions of porous calcite (tufa limesto$n e)$ in the bed of spring runs (rheocrenes) and modest $(\leq 1.5$ $\mathrm{m}$ high) mound-like limestone formations formed in small 'waterfall/creek' systems (hygropetric rheocrenes; Figure 1). Several types may occur at the same site, e.g. water from a hygropetric spring may join seepage of one or more rheohelocrenes within a single source area and transitional situations are common. Most spring waters are moderately alkaline (interquartile $\mathrm{pH}$ range $7.4-8$ ), with $\mathrm{pH}$ typically increasing slightly downstream. $\mathrm{EC}_{25}$ values usually range between 714 and $950 \mu \mathrm{S} . \mathrm{cm}^{-1}$ and alkalinities from 4.9 to 6.6 meq. $\mathrm{l}^{-1}$ (Oosterlynck \& De Bie in press). Bicarbonate and calcium predominate with levels (upper and lower interquartile ranges 300-403 and 133-169 mg..$^{-1}$, respectively; OOSTERLYNCK $\&$ DE BIE in press) that are well above the minimum for carbonate-depositing systems (PENTECost 2005). Water chemistry varies somewhat with the nature of the Tertiary aquifer and therefore geographically, but overall such differences remain limited. Seasonal differences also occur, e.g. in calcium and ammonium. In some instances, elevated levels of ammonium, nitrate, sodium, potassium, chloride and sulphate suggest pollution. Nitrate leaching to the phreatic groundwater is generally quite considerable in southern Flenders (EPPINGER \& THOMAS 2007), with atmospheric deposition (usually 21-35 kg N ha ${ }^{-1}$ year $^{-1}$; Overloop et al. 2011) and agriculture as principal sources. Palustriella commutata (Hedw.) OcHYRA, and, quite occasionally, Eucladium verticillatum (BRIDEL) BRUCH et SCHIMPER are the more characteristic mosses representing the Cratoneurion in the stricter sense. These are usually accompanied by Brachythecium rivulare ScHIMPER, Cratoneuron filicinum (Hedw.) SPRUcE, Oxyrrhynchium hians (Hedw.) Loeske, Pellia endiviifolia (Dicks) Dumort. and Plagiomnium undulatum (HeDw.) T.J. KoP.

\section{Material AND Methods}

Samples were collected from thirteen springs (Figure 2) representing seven of the ten aquifers in Flanders that give rise to notable carbonate precipitation (Table 1). Only three aquifers were represented by more than one spring (Tielt 1 , Vaals and Brusseliaan). Altitude varied from c. 30 to $200 \mathrm{~m}$. In some cases, the source was confined by tubing or masonry. One of the sites, MOEN, was completely artificial, resulting from cutting a canal through the crest of a hill in the 1970's. Soils consisted of loam, sandy loam or occasionally, fen wood peat. All investigated springs, except for one, were under deciduous forest cover, usually dominated by Fraxinus excelsior L. and Carpinus betulus L. (Carici remotae-Fraxinetum, Carpinion).

Sampling sites were attributed to major spring types according to their local character (Table 1). In six springs, samples were positioned differently relative to the area of calcite formation. This was categorized as: (a) at the source, (b) just in front of the stretch with carbonate precipitation, or (c) within the main precipitation zone. Distance between the source and more downstream samples varied. Most sites 


\begin{tabular}{|c|c|c|c|c|c|c|c|c|c|c|c|c|c|c|c|c|c|c|c|c|c|c|c|c|}
\hline water samples & m & + & + & + & $m$ & $N$ & m & m & + & + & m & $\sim$ & $m$ & m & m & + & m & - & m & m & & & & \\
\hline $\begin{array}{l}\mathrm{NH}_{4}^{+}-\mathrm{N} \\
\left(\mathrm{mg} . \mathrm{l}^{-1}\right)\end{array}$ & $\begin{array}{l}\circ \\
: \\
0\end{array}$ & : & ôें & $\begin{array}{l}\overrightarrow{0} \\
\dot{0} \\
\dot{0}\end{array}$ & हैं & हैं & ôे & o̊ & के & $\stackrel{\vec{o}}{\circ}$ & ồ & के & 号 & $\vec{n}$ & $\hat{0}$ & $\vec{n}$ & है. & हैं & 党 & $\stackrel{\vec{\sigma}}{\circ}$ & 虫 & : & ఏ & - \\
\hline $\begin{array}{l}\mathrm{NO}_{3}^{-}-\mathrm{N} \\
\left(\mathrm{mg} \cdot \mathrm{l}^{-1}\right)\end{array}$ & $\stackrel{0}{0}$ & $\begin{array}{l}\vec{\infty} \\
\stackrel{\bigcup}{\bigcup}\end{array}$ & 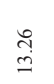 & $\stackrel{\substack{? \\
=}}{=}$ & $\begin{array}{l}: \\
\stackrel{n}{0} \\
\stackrel{0}{0}\end{array}$ & ڤે & $\stackrel{\vec{i}}{i}$ & $\hat{n}$ & 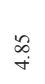 & $\stackrel{R}{2}$ & $\overrightarrow{3}$ & $\stackrel{\infty}{\sim}$ & $\stackrel{\vec{n}}{\stackrel{n}{3}}$ & İ & $\overrightarrow{\vec{j}^{\prime}}$ & $\vec{n}$ & $\stackrel{0}{0}$ & $\stackrel{\tilde{O}}{0}$ & $\stackrel{n}{=}$ & $\stackrel{\circ}{\stackrel{2}{2}}$ & $\begin{array}{l}\grave{b} \\
\infty\end{array}$ & $\stackrel{n}{n}$ & 3 & - \\
\hline $\begin{array}{l}\text { SRP } \\
\left(\mu \mathrm{g} . \mathrm{I}^{-1}\right)\end{array}$ & ल & ్ㅗ & $\infty$ & $=$ & $\simeq$ & $\cong$ & ले & $\infty$ & $\infty$ & $\infty$ & $\infty$ & $\infty$ & 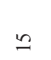 & $\infty$ & in & $\hat{\sim}$ & $\infty$ & $\infty$ & ส & $\infty$ & 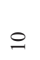 & $\stackrel{\circ}{\simeq}$ & $\infty$ & I \\
\hline $\begin{array}{l}\mathrm{Fe}^{2 / 3+} \\
\left(\mathrm{mg} \cdot \mathrm{l}^{-1}\right)\end{array}$ & 菅 & $\stackrel{\leftrightarrow}{\circ}$ & $\stackrel{n}{\dot{\varphi}}$ & $\stackrel{\leftrightarrow}{\dot{v}}$ & $\stackrel{n}{\dot{\theta}}$ & $\stackrel{n}{\dot{\theta}}$ & $\stackrel{\leftrightarrow}{\circ}$ & $\stackrel{n}{\dot{\theta}}$ & $\ddot{\leftrightarrow}$ & $\ddot{\leftrightarrow}$ & $\stackrel{\ddot{O}}{\dot{\theta}}$ & 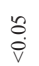 & $\stackrel{\leftrightarrow}{\dot{b}}$ & $\stackrel{n}{\dot{\theta}}$ & $\stackrel{\leftrightarrow}{\circ}$ & $\stackrel{\leftrightarrow}{\dot{v}}$ & $\stackrel{1}{0}$ & $\ddot{\dot{\varphi}}$ & $\stackrel{n}{\dot{v}}$ & $\stackrel{n}{\dot{v}}$ & $\ddot{\dot{v}}$ & $\stackrel{\leftrightarrow}{\dot{\theta}}$ & & 1 \\
\hline $\begin{array}{l}\mathrm{Na}^{+} \\
\left(\mathbf{m g} . \mathrm{l}^{-1}\right)\end{array}$ & $\exists$ & $\stackrel{\leftrightarrow}{\stackrel{\leftrightarrow}{\longrightarrow}}$ & $\stackrel{\infty}{\stackrel{\infty}{\jmath}}$ & Ðِ & $\stackrel{g}{\underline{b}}$ & $\stackrel{\infty}{\stackrel{\infty}{\oplus}}$ & $\stackrel{\tilde{m}}{\sim}$ & $\stackrel{\tilde{3}}{\widetilde{3}}$ & $\stackrel{\circlearrowright}{\text { İ }}$ & $\stackrel{\tilde{\mathrm{I}}}{\underline{\mathrm{N}}}$ & $\stackrel{2}{2}$ & $\hat{\sigma}$ & 3 & $\overrightarrow{6}$ & $\stackrel{\ddot{n}}{\mathrm{i}}$ & $\ddot{n}$ & $\stackrel{\infty}{\sim}$ & $\underset{\sim}{\sim}$ & $\stackrel{9}{=}$ & فे & Iे & $\stackrel{\circlearrowright}{\text { Ij }}$ & in & - \\
\hline $\begin{array}{l}\mathbf{M g}^{2+} \\
\left(\mathbf{m g} . \mathrm{l}^{-1}\right)\end{array}$ & $\stackrel{\circ}{\stackrel{0}{\Xi}}$ & $\stackrel{\circ}{\infty}$ & $\hat{n}$ & $\stackrel{\infty}{\sigma}$ & $\stackrel{t}{\sigma}$ & $\vec{\infty}$ & בิ & $\stackrel{0}{\circ}$ & $\overrightarrow{\underline{n}}$ & $\stackrel{2}{a}$ & $\hat{i}$ & $\stackrel{\circ}{i}$ & 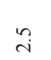 & สี & $\ddot{r}$ & $\stackrel{\circ}{i}$ & $\underset{\mathcal{I}}{\mathcal{I}}$ & 我 & $\stackrel{3}{\varrho}$ & 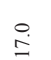 & Iี & $\stackrel{\infty}{=}$ & ก & - \\
\hline $\begin{array}{l}\mathbf{K}^{+} \\
\left(\mathbf{m g} . \mathrm{l}^{-1}\right)\end{array}$ & กู & $\vec{i}$ & $\stackrel{\circ}{i}$ & $\stackrel{\infty}{i}$ & $\stackrel{n}{m}$ & $\dot{m}$ & $\stackrel{\vec{n}}{\mathrm{n}}$ & $\stackrel{m}{+}$ & $\stackrel{m}{m}$ & $\stackrel{n}{-}$ & $\hat{i}$ & $ક$ & $\stackrel{\circ}{-}$ & $\stackrel{\circ}{-}$ & $\stackrel{\circ}{-}$ & $n$ & in & $\stackrel{n}{\sim}$ & $\stackrel{I}{I}$ & 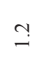 & ì & ले & is & 1 \\
\hline $\begin{array}{l}\mathrm{Ca}^{2^{+}} \\
\left(\mathrm{mg} \cdot \mathrm{l}^{-1}\right)\end{array}$ & 产 & 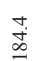 & $\stackrel{+}{\stackrel{9}{9}}$ & $\underset{\substack{\infty \\
\curvearrowleft}}{\curvearrowleft}$ & $\stackrel{m}{\stackrel{m}{2}}$ & 字 & $\begin{array}{l}0 \\
\dot{0} \\
\dot{g}\end{array}$ & 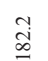 & $\stackrel{+}{\stackrel{\Xi}{E}}$ & $\begin{array}{l}\infty \\
\stackrel{\infty}{\varrho}\end{array}$ & $\underset{\substack{+g}}{+}$ & $\begin{array}{l}\infty \\
\ddot{n} \\
\Leftrightarrow\end{array}$ & 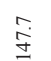 & $\begin{array}{l}\stackrel{\circlearrowright}{\mathrm{j}} \\
\mathrm{J}\end{array}$ & 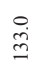 & $\vec{\infty}$ & $\overrightarrow{\vec{a}}$ & 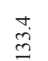 & $\overbrace{n}^{n}$ & $\begin{array}{l}0 \\
\stackrel{0}{0} \\
\stackrel{n}{n}\end{array}$ & $\frac{3}{4}$ & 品 & $\hat{\imath}$ & - \\
\hline $\begin{array}{l}\mathrm{Cl}_{(\mathbf{m g}}^{-} \\
\left.\mathrm{l}^{-1}\right)\end{array}$ & $\stackrel{m}{\infty}$ & $\vec{n}$ & ํ. & के & $\begin{array}{l}\infty \\
\underset{j}{j}\end{array}$ & $\stackrel{F}{\dot{F}}$ & $\underset{\sim}{\stackrel{\sim}{\sim}}$ & $\stackrel{\infty}{\infty}$ & $\stackrel{i}{m}$ & $\begin{array}{l}\infty \\
\stackrel{0}{0}\end{array}$ & $\stackrel{m}{d}$ & $\stackrel{\infty}{\circ}$ & ฮั & $\stackrel{+}{\check{a}}$ & $\stackrel{3}{-3}$ & $\stackrel{3}{2}$ & fó & $\vec{y}$ & $\underset{j}{\infty}$ & i্j & $\vec{i}$ & లై & ster & - \\
\hline $\begin{array}{l}\mathrm{SO}_{4}^{2-} \\
\left(\mathbf{m g}^{-1}\right)\end{array}$ & $\vec{g}$ & $\grave{\&}$ & กู & $\overbrace{\infty}^{\infty}$ & ㅇ. & ณू. & 迥 & กั่ & $\ddot{8}$ & ตू. & 字 & के & 过 & $\vec{\sigma}$ & $\underset{\infty}{\mathbb{i}}$ & $\begin{array}{l}b \\
\stackrel{i}{N}\end{array}$ & $\stackrel{\substack{n \\
\infty}}{\infty}$ & 8 & $\underset{\infty}{\infty}$ & fi & $\underset{\infty}{\infty}$ & $\stackrel{\dot{0}}{\infty}$ & 7 & 1 \\
\hline $\begin{array}{l}\mathrm{HCO}_{-}^{-} \\
\left(\mathrm{mg}^{-\mathrm{I}^{-1}}\right)\end{array}$ & 羍 & $\infty$ & : & f & $\frac{n}{m}$ & $\equiv$ & ஓ & $\tilde{y}$ & ஓे & $\stackrel{\infty}{\infty}$ & m & $\frac{n}{m}$ & $\approx$ & 。్ల & : n & $\vec{a}$ & ₹ี & ঃ్ల & 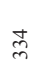 & $\stackrel{0}{\neq}$ & $\varlimsup_{\infty}^{\infty}$ & gे & is & - \\
\hline $\begin{array}{l}\text { ALK } \\
\left.\text { (eq. } \mathrm{H}^{-1}\right)\end{array}$ & $\stackrel{0}{0}_{0}^{\circ}$ & 3 & iे & $\ddot{\circ}$ & กี่ & $\vec{n}$ & $\hat{\sigma}$ & oे & $\hat{\sigma}$ & ţ. & $n$ & ถู & 3 & ir & $\stackrel{+}{+}$ & $\stackrel{\infty}{\infty}$ & aे & $g$ & 古 & $\stackrel{\infty}{\infty}$ & $n$ & in & ? & - \\
\hline $\begin{array}{l}\mathrm{EC}_{25} \\
\left(\mu \mathrm{S} . \mathrm{cm}^{-1}\right)\end{array}$ & $\stackrel{\infty}{\infty}$ & $\approx$ & $\mathbb{J}$ & ठे & fี & $\vec{\infty}$ & $\bar{\varnothing}$ & $\kappa$ & $\%$ & ळ & $\ddot{8}$ & $\stackrel{2}{2}$ & $\stackrel{2}{2}$ & 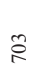 & 。્రి & $\stackrel{0}{0}$ & $\stackrel{n}{\circ}$ & $\preccurlyeq$ & $\stackrel{m}{2}$ & రి & $\underset{\infty}{\forall}$ & $\infty_{\infty}^{t}$ & 3 & - \\
\hline $\mathrm{pH}$ & $\stackrel{2}{ }$ & 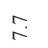 & $\stackrel{2}{2}$ & 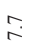 & $\stackrel{\infty}{\infty}$ & $\vec{\infty}$ & $\stackrel{9}{r}$ & $\stackrel{\infty}{\sim}$ & $\stackrel{\circ}{\infty}$ & $\stackrel{?}{r}$ & $\stackrel{\infty}{\stackrel{\infty}{\Gamma}}$ & $\stackrel{\infty}{\stackrel{\infty}{\sim}}$ & $\stackrel{n}{n}$ & $\stackrel{2}{r}$ & $\stackrel{\circ}{r}$ & $\stackrel{a}{r}$ & $\stackrel{m}{r}$ & ${ }_{\infty}^{0}$ & $\vec{\infty}$ & $\stackrel{ }{r}$ & $\stackrel{\infty}{\sim}$ & I & c & I \\
\hline shade & N & in & + & + & + & + & m & in & in & m & in & + & in & m & n & in & $\sim$ & - & in & in & + & I & 1 & 1 \\
\hline tufa & ナ & m & in & + & in & in & $m$ & 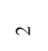 & $m$ & N & n & + & m & $n$ & $\sim$ & in & $m$ & $m$ & n & - & $m$ & I & s & 1 \\
\hline $\begin{array}{l}\text { total cover } \\
(\%)\end{array}$ & $\infty$ & 8 & in & q & 8 & R & $尺$ & 8 & ల్ల & i & 8 & in & 8 & 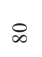 & $q$ & 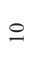 & 8 & q & 8 & 8 & 8 & $\stackrel{\infty}{n}$ & a & $\sigma$ \\
\hline $\begin{array}{l}\text { bryophyte cover } \\
\text { (\%) }\end{array}$ & ¿ & in & in & q & त्र & సి & q & in & in & సి & ণి & in & in & in & 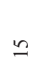 & $\subseteq$ & $\triangleright$ & in & $m$ & 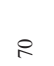 & $\underset{\infty}{\infty}$ & $m$ & ๙ & $\approx$ \\
\hline $\begin{array}{l}\text { altitude } \\
\text { (m TAW) }\end{array}$ & 8 & in & $\approx$ & A & $\pi$ & 5 & 5 & f & q & 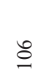 & 志 & $\Leftrightarrow$ & $\stackrel{\infty}{\sim}$ & 专 & ฮิ & פ & $g$ & ते & $m$ & 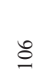 & $\approx$ & Lू & is & \\
\hline forest/fen type & 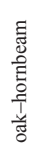 & $\begin{array}{l}\bar{y} \\
\frac{\bar{v}}{\pi} \\
\frac{\pi}{5}\end{array}$ & $\begin{array}{l}\frac{\vec{v}}{\bar{v}} \\
\frac{1}{\bar{v}} \\
\frac{w}{w}\end{array}$ & $\begin{array}{l}\frac{\vec{s}}{\frac{\pi}{\pi}} \\
\frac{L}{\tilde{s}}\end{array}$ & 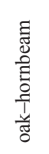 & 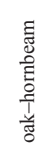 & 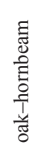 & $\begin{array}{l}\frac{\vec{y}}{\bar{y}} \\
\frac{\vec{n}}{\bar{y}}\end{array}$ & $\begin{array}{l}\frac{\vec{g}}{\frac{\pi}{\pi}} \\
\frac{\vec{s}}{\tilde{g}}\end{array}$ & 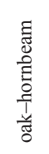 & $\frac{\frac{\tilde{g}}{\frac{\pi}{\pi}}}{\frac{\bar{t}}{\bar{v}}}$ & $\begin{array}{l}\frac{\vec{g}}{\bar{v}} \\
\frac{\underline{v}}{\bar{v}}\end{array}$ & $\begin{array}{l}\frac{\overrightarrow{\frac{v}{\pi}}}{\frac{\pi}{\pi}} \\
\frac{1}{\frac{5}{\pi}}\end{array}$ & $\begin{array}{l}\frac{\vec{s}}{\frac{\pi}{\pi}} \\
\frac{L}{\tilde{g}}\end{array}$ & $\begin{array}{l}\frac{\vec{g}}{\bar{v}} \\
\frac{L}{\bar{v}}\end{array}$ & $\begin{array}{l}\frac{\vec{v}}{\frac{\pi}{\pi}} \\
\frac{\vec{L}}{w}\end{array}$ & $\begin{array}{l}\frac{\vec{v}}{\bar{v}} \\
\frac{5}{\tilde{n}}\end{array}$ & 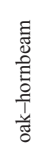 & 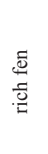 & $\begin{array}{l}\frac{\vec{v}}{\bar{v}} \\
\frac{L}{\frac{w}{w}}\end{array}$ & & & & \\
\hline soil & 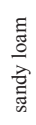 & 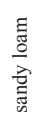 & 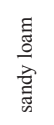 & 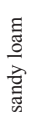 & 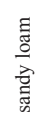 & 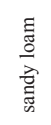 & 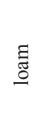 & 镸 & 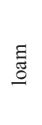 & 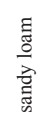 & $\stackrel{\Xi}{\underline{\Xi}}$ & 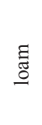 & ळ & 苑 & 镸 & Е & 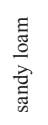 & 镸 & 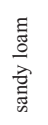 & $\stackrel{\Xi}{\Xi}$ & & & & \\
\hline spring type & 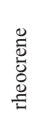 & 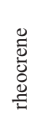 & 일 & 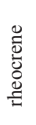 & 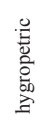 & 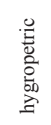 & 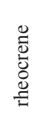 & 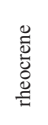 & 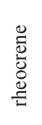 & 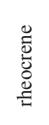 & $\begin{array}{l}\frac{\partial}{2} \\
\frac{0}{2} \\
\frac{0}{2} \\
\frac{8}{2}\end{array}$ & 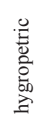 & 힐 & 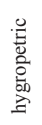 & 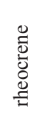 & 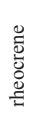 & 递 & 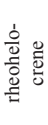 & 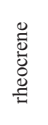 & 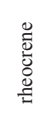 & & & & \\
\hline aquifer & $\stackrel{\Xi}{\stackrel{\Xi}{\Xi}}$ & $\underset{\Xi}{\Xi}$ & 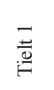 & 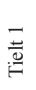 & 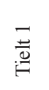 & 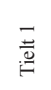 & 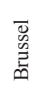 & 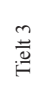 & 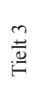 & 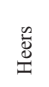 & 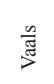 & 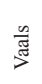 & 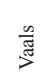 & 总 & 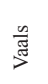 & 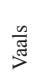 & $\underset{\Xi}{\stackrel{E}{E}}$ & $\sum_{\grave{\Sigma}}^{\bar{\Xi}}$ & $\begin{array}{l}\text {.्. } \\
\text { 邑 } \\
\text { 邑 }\end{array}$ & 定 & & & & \\
\hline $\begin{array}{l}\text { longitude } \\
\left({ }^{\circ}\right)\end{array}$ & 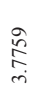 & 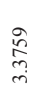 & 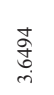 & 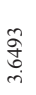 & 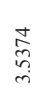 & 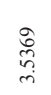 & $\begin{array}{l}\underset{+}{+} \\
\stackrel{+}{+} \\
\stackrel{+}{*}\end{array}$ & $\underset{\substack{+\dot{r}}}{\frac{\vec{t}}{2}}$ & 啇 & 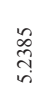 & $\begin{array}{l}\text { Dे } \\
\infty \\
\text { in }\end{array}$ & 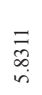 & $\begin{array}{l}\underset{d}{d} \\
\underset{\infty}{\infty} \\
i\end{array}$ & 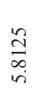 & $\begin{array}{l}\stackrel{m}{a} \\
\stackrel{a}{a}\end{array}$ & $\begin{array}{l}\stackrel{n}{a} \\
\stackrel{a}{ } \\
i n\end{array}$ & $\begin{array}{l}\tilde{b} \\
\dot{0} \\
\dot{r}\end{array}$ & 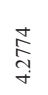 & 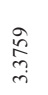 & 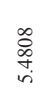 & & & & \\
\hline $\begin{array}{l}\text { latitude } \\
\left({ }^{\circ}\right)\end{array}$ & 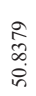 & $\begin{array}{l}\infty \\
\infty \\
\infty \\
\infty \\
\infty \\
0\end{array}$ & $\begin{array}{l}\tilde{n} \\
\infty \\
\infty \\
\dot{p}\end{array}$ & 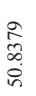 & 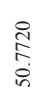 & $\begin{array}{l}\vec{N} \\
\hat{D} \\
\stackrel{n}{n}\end{array}$ & 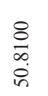 & $\begin{array}{l}\text { त̂ } \\
\stackrel{0}{0} \\
\text { in }\end{array}$ & $\begin{array}{l}\overline{0} \\
\therefore \\
\dot{n}\end{array}$ & $\begin{array}{l}\stackrel{\vec{N}}{\hat{0}} \\
\dot{i n}\end{array}$ & 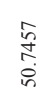 & 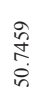 & $\begin{array}{l}\infty \\
\stackrel{0}{0} \\
\hat{n} \\
\dot{p}\end{array}$ & $\begin{array}{l}\frac{m}{n} \\
\hat{n} \\
\dot{n}\end{array}$ & 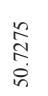 & 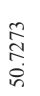 & 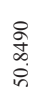 & 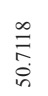 & $\begin{array}{l}+ \\
\stackrel{+}{\infty} \\
\stackrel{0}{\infty}\end{array}$ & $\begin{array}{l}\tilde{b} \\
\stackrel{0}{\infty} \\
\dot{D}\end{array}$ & & & & \\
\hline sample & 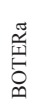 & 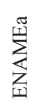 & $\begin{array}{l}\text { 道 } \\
\text { 畜 } \\
\text { 畜 }\end{array}$ & 䕎 & 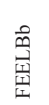 & 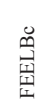 & $\begin{array}{l}\text { 兽 } \\
\text { 总 }\end{array}$ & 离 & 离 & 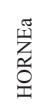 & $\underset{\tilde{\Xi}}{\stackrel{\tilde{y}}{\tilde{y}}}$ & $\frac{\hat{\tilde{Z}}}{\underline{a}}$ & 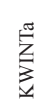 & $\underset{\underline{z}}{\vec{z}}$ & 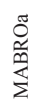 & 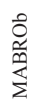 & $\begin{array}{l}\text { 覓 } \\
\stackrel{2}{\Sigma}\end{array}$ & $\begin{array}{l}\sum_{\mathbf{1}}^{0} \\
\stackrel{0}{0}\end{array}$ & $\begin{array}{l}\stackrel{\ddot{i}}{\mathbf{i}} \\
\text { 点 }\end{array}$ & 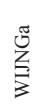 & 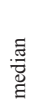 & 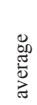 & & \\
\hline
\end{tabular}


Table 2. Significant Pearson or Spearman correlations between environmental variables for all examined sites $(\mathrm{p} \leq 0.05, \mathrm{p} \leq 0.01, \mathrm{p} \leq 0.001)$.

\begin{tabular}{lccccccccccc}
\hline & $\begin{array}{c}\text { plant } \\
\text { cover }\end{array}$ & $\mathbf{p H}$ & $\begin{array}{c}\text { alkali- } \\
\text { nity }\end{array}$ & $\mathbf{S O}_{4}^{2-}$ & $\mathbf{C l}^{-}$ & $\mathbf{K}^{+}$ & $\mathbf{M g}^{2+}$ & $\mathbf{C a}^{2+}$ & $\mathbf{N a}^{+}$ & $\mathbf{N O}_{3}^{-}-\mathbf{N}$ & $\mathbf{N H}_{4}^{+}-\mathbf{N}$ \\
\hline bryophyte cover & 0.61 & - & 0.46 & - & - & - & - & 0.52 & - & - & - \\
tufa & & 0.50 & -0.47 & - & - & - & - & - & - & - & -0.54 \\
shade & & & - & - & - & -0.54 & - & - & - & - & - \\
$\mathrm{EC}_{25}$ & & & $\mathbf{0 . 7 0}$ & $\mathbf{0 . 7 1}$ & $\mathbf{0 . 8 8}$ & - & $\mathbf{0 . 7 9}$ & $\mathbf{0 . 8 4}$ & $\mathbf{0 . 7 3}$ & - & - \\
alkalinity & & & & - & - & - & 0.64 & $\mathbf{0 . 7 4}$ & - & - & - \\
$\mathrm{SO}_{4}{ }^{2-}$ & & & & & 0.57 & $\mathbf{0 . 7 2}$ & 0.62 & - & $\mathbf{0 . 7 8}$ & -0.54 & - \\
$\mathrm{Cl}^{-}$ & & & & & & - & 0.64 & $\mathbf{0 . 6 9}$ & $\mathbf{0 . 7 0}$ & - & - \\
$\mathrm{K}^{+}$ & & & & & & - & 0.55 & - & $\mathbf{0 . 6 9}$ & -0.53 & - \\
$\mathrm{Mg}^{2+}$ & & & & & & & & - & $\mathbf{0 . 7 3}$ & - & - \\
\hline
\end{tabular}

were sampled two- or three-monthly for hydrochemistry from spring to autumn in a single year, but data were more limited for others (Table 1). Water samples were transported to the laboratory in firmly closed vials at $4{ }^{\circ} \mathrm{C}$ and analysed for $\mathrm{pH}$, electrical conductivity (EC, WTW-multimeter), alkalinity (titration to $\mathrm{pH} 4.50$ and 8.35), major ions and nutrients (calcium, magnesium, sodium, potassium, sulphate, chloride, soluble reactive phosphorus - SRP, nitrate and ammonium by ion chromatography with a Dionex ICS-3000) and total iron (inductively coupled plasma atomic emission spectrophotometry with Agilent Liberty II) according to standard protocols. EC and $\mathrm{pH}$ were also measured on site, but field readings were only retained for EC. The amount of tufa limestone was judged on a scale from 0 (none) to 5 (massive). The degree of shading in summer was also estimated ordinally from 0 (none) to 5 (full).

Only diatom assemblages from non-living substrates were analysed. Samples consisted of sediment (usually sand with some fine organic matter), carbonate concretions and pebbles from the bed of runs, or scrapings of the surface of more massive limestone formations. All diatom samples were taken on 16 and 17 October 2011. In the Alps, CANTONATI et al. (2012b) considered a single sample at the end of the vegetation season sufficient for assessing assemblage structure and typological differences.

Diatom samples were cleaned with concentrated hydrogen peroxide at $70{ }^{\circ} \mathrm{C}$ and carbonates were dissolved with hydrochloric acid. Strewn mounts $(36 \times 24 \mathrm{~mm})$ were prepared using Naphrax ${ }^{\circledR}$ and examined with an Olympus BX 51 microscope equipped with differential interference optics. Up to 500 valves were counted along random transects at high magnification to calculate relative percentage abundances and additional taxa were identified by further screening the slides.

In addition to standard floras and monographs (e.g. REICHARDT 1999; KRAMMER 1997a, 1997b, 2000, 2002, 2003; Krammer \& Lange-Bertalot 1999-2004; Lange-Bertalot 2001; LANGE-Bertalot et al. 2003; LeVKov 2009, LeVKov et al. 2013), various thematic papers were used for identification.

Indicator Species Analysis (DUfRĖNE \& LEGENDRE 1997) was used to identify characteristic taxa of predefined groups of samples. Similarity between assemblages was examined by cluster analysis (Ward's method with Euclidian distances of square-root transformed abundances). Detrended Correspondence Analysis (DCA) was used to analyse species turnover and (constrained) Redundancy Analysis (RDA) with forward selection to elucidate the relation between assemblage composition and environmental variables. Only taxa with at least $1 \%$ relative abundance in one sample were included in ordinations with square-root transformation and down-weighting of rare taxa. Some environmental variables were transformed as $\log _{10}$ and percentages as arcsine square root (Table 1). Samples were weighted proportionally by the ratio of their valve count to the standard total of 500 valves. Analyses were carried out using PC-ORD for Windows (MCCune \& MEFord 1999) and CANOCO for Windows (TER BraAK \& Smilauer 2003).

The 'Indice de Polluosensibilité' (IPS; CEMAGREF 1982), 'Trophie Index' (TI ${ }_{\mathrm{DIA}}$; RotT el al. 1999) and relative abundance of taxa linked to anthropogenic alteration of springs in the marl-loess region of Hessen (\% A; WERUM \& LANGE-BertALOT 2004, p. 103) were calculated, as well as the number $(\#)$ and percentage (\%) of taxa considered endangered (E; categories 1, 2, 3 and $\mathrm{G}$ ) or rare (R; categories $\mathrm{R}$ and unrecorded in Germany) on the Red List for Germany and Central Europe (Lange-Bertalot \& Steindorf 1996; Cantonati \& Lange-Bertalot 2010). Some more recently described taxa with very few records were added to the group of rare taxa (Table S1). The most xerotolerant taxa (X) were selected mainly from compilations by DENYS (1991) and VAN DAM et al. (1994). Differences between groups of samples were explored by means of Kruskal-Wallis Rank Sum tests and correlations between variables by the Pearson coefficient or, if one or both were ordinal, Spearman's rho. These tests were done with Tibco Spotfire S+ 8.2 for Windows.

\section{Results}

Conductivity, $\mathrm{pH}$, alkalinity and calcium showed relatively little variation (Table 1). Iron content was always low. Nitrate-nitrogen was often higher than what would be expected in pristine springs, remaining below measurable concentrations only for BOTER, MATER 
Table 3. Characteristics and cluster attribution of diatom assemblages from springs with Cratoneurion in Flanders. FONTb and WIJNGa not considered in averages. \% relative abundance, \# number of taxa; A: indicators of anthropogenic alteration; R: Red List group rare; E: Red List group endangered; X: xerotolerant (see text).

\begin{tabular}{|c|c|c|c|c|c|c|c|c|c|c|}
\hline sample & $\operatorname{taxa}$ & $\# \mathbf{R}$ & $\% \mathbf{R}$ & $\# \mathbf{E}$ & $\% \mathrm{E}$ & $\% \mathrm{X}$ & IPS & $\mathbf{T I}_{\text {DIA }}$ & $\% \mathrm{~A}$ & group \\
\hline BOTERa & 72 & 9 & 4.4 & 9 & 2.6 & 21.0 & 16.6 & 2.7 & 3.4 & I \\
\hline ENAMEa & 64 & 11 & 8.8 & 5 & 1.8 & 62.2 & 16.0 & 2.8 & 0.0 & III \\
\hline ENAMEb & 61 & 6 & 2.0 & 7 & 2.8 & 18.8 & 17.0 & 2.3 & 1.4 & II \\
\hline FEELBa & 50 & 5 & 1.4 & 3 & 1.0 & 20.0 & 16.2 & 2.5 & 1.8 & II \\
\hline FEELBb & 57 & 6 & 3.4 & 5 & 0.8 & 21.8 & 15.6 & 2.7 & 1.6 & II \\
\hline FEELBc & 64 & 4 & 3.2 & 5 & 0.8 & 17.4 & 16.0 & 2.7 & 0.8 & II \\
\hline FONTb & 2 & 1 & - & 0 & - & - & - & - & - & distinct \\
\hline GALMAa & 85 & 10 & 3.0 & 10 & 7.2 & 31.4 & 16.0 & 2.5 & 2.6 & I \\
\hline GALMAb & 88 & 9 & 4.0 & 12 & 7.4 & 30.0 & 16.2 & 2.8 & 0.8 & I \\
\hline HORNEa & 53 & 8 & 12.8 & 6 & 2.8 & 38.6 & 15.0 & 2.8 & 0.2 & I \\
\hline KRINDa & 64 & 4 & 3.2 & 11 & 7.0 & 51.2 & 16.9 & 1.9 & 0.2 & III \\
\hline KRINDb & 63 & 3 & 1.0 & 4 & 1.2 & 30.4 & 16.7 & 2.2 & 0.5 & III \\
\hline KWINTa & 70 & 7 & 9.2 & 8 & 4.6 & 1 & 16.0 & 2.5 & 0.6 & III \\
\hline KWINTb & 47 & 5 & 0.8 & 4 & 0.6 & 58.0 & 17.9 & 2.3 & 0.2 & II \\
\hline MABROa & 76 & 11 & 5.4 & 9 & 6.6 & 58.0 & 15.6 & 2.7 & 1.0 & I \\
\hline MABROb & 67 & 4 & 3.1 & 3 & 3.1 & 45.1 & 15.7 & 2.3 & 1.6 & III \\
\hline MATERb & 89 & 10 & 3.0 & 8 & 4.0 & 24.6 & 13.9 & 2.9 & 23.1 & I \\
\hline MOENb & 27 & 5 & 15.0 & 4 & 5.6 & 37.6 & 15.4 & 2.7 & 0.0 & distinct \\
\hline STEENc & 49 & 6 & 1.8 & 5 & 0.6 & 36.8 & 15.2 & 2.5 & 1.6 & II \\
\hline WIJNGa & 15 & 2 & - & 2 & - & - & - & - & - & distinct \\
\hline mean $\pm \mathrm{SD}$ & $\begin{array}{c}63.6 \pm \\
15.6\end{array}$ & $\begin{array}{c}6.8 \pm \\
2.6\end{array}$ & $\begin{array}{c}4.8 \pm \\
4.1\end{array}$ & $6.6 \pm 2.8$ & $3.4 \pm 2.5$ & $33.5 \pm 16.5$ & $16.0 \pm 0.9$ & $2.6 \pm 0.3$ & $2.3 \pm 5.3$ & - \\
\hline
\end{tabular}

and MOEN and exceeding $10 \mathrm{mg} \cdot \mathrm{l}^{-1}$ in ENAME, FEELB and KWINT. Correlations between environmental variables for the sampled sites did not deviate from Flemish carbonate springs in general. Highly significant coefficients occurred mainly among EC, chloride, calcium, magnesium, sodium, sulphate and potassium (Table 2). Potassium also related inversely to shade, whilst ammonium tended to be lower where more calcite occurred. Nitrate correlated negatively to potassium and sulphate but varied independently from SRP.

In total, c. 310 diatom morphotaxa were recorded in the 20 samples (Table S1, with some illustrated in Figures 3-5). Almost half of them occurred in only one sample (Figure 6). Most taxa belonged to Pinnularia (40), followed by Gomphonema (27), Navicula s.s. (24), Nitzschia (19) and Stauroneis (16). The most constant species were Halamphora normanii, Stauroneis thermicola (90\% of all samples), Achnanthidium jackii (Figure 3 a), Navicula cincta, Planothidium lanceolatum (85\%), Achnanthidium minutissimum, Humidophi- la brekkaensis (Figure $3 \mathrm{u}$ ), Nitzschia linearis, Planothidium frequentissimum (80\%), Amphora pediculus (Figure 3 c), Eolimna tantula (Figure 3 h), Stauroneis parathermicola (Figure $5 \mathrm{f}-\mathrm{g}$ ) and Surirella terricola (75\%). Some 36 taxa, mostly represented by fragments or very few valves, could not be identified confidently to species level. A few of them probably still require formal description.

Two samples contained extremely few and mostly corroded diatom valves. Extensive searching yielded only Stauroneis muriella (Figure $5 \mathrm{c}-\mathrm{e}$ ) and $S$. thermicola with 2 valves each in FONTEb. Merely 27 valves and 15 taxa could be counted from several slides of WIJNGa. Valve concentration was also very low for MABROb (total count 193 valves), but with 67 taxa, species richness was more in line with that of the remaining samples. Neglecting FONTEb and WIJN$\mathrm{Ga}$, the average number of taxa per sample was 64 , the highest number (89) occurring in MATERb (Table 3). On average 57 taxa were noted in hygropetric rheo- 
crenes, increasing to 67 in rheocrenes, though differences in richness between geomorphological types were not significant (Table 4).

Several of the observed taxa were recorded rarely so far, and often mainly from other springs, e.g. Cymbopleura diminuta, C. subaustriaca, Humidophila irata, Frustulia amosseana, Gomphonema extentum, Navicula arctotenelloides (Figure 3 w), Navicula s.l. harderi (Figure 3 ae), N. s.l. obsoleta (Figure $3 \mathrm{ag}$ ), $N$. s.l. parsura, Navicula s.1. subseminulum (Figure 3 ah), Neidium minutissimum, Nitzschia harderi (Figure 4 c-d), Pinnularia frauenbergiana var. caloneiopsis (Figure $4 \mathrm{f}$ ) and Pulchella minutissima (Figure $4 \mathrm{v}$ ). Almost $14 \%$ of all taxa (43) was listed as rare and $11 \%$ (34) as endangered. On average there were 6.8 rare and 6.6 endangered taxa per sample (Table 3 ), both categories together representing $21.3 \pm 5.8 \%$ of all taxa in a sample. The relative abundance of RL taxa usually remained quite modest $(8.4 \pm 5.3 \%)$. GALMAa, GALMAb, KWINTa and MABROa consistently scored above average for number as well as abundance of RL taxa. Most RL metrics also scored better than average for MATERb, in spite of the eutrophic character of its assemblage according to $\mathrm{TI}_{\mathrm{DIA}}$ (Table 3 ). Interestingly the relative abundance of rare taxa was also more substantial in MOENb. The representation of RL taxa was lowest in hygropetric springs, but differences were again insignificant (Table 4).

The relative abundance of xerotolerant taxa varied strongly. KWINTa hardly contained any (1\%), whereas they predominated in ENAMEa, KRINDa, KWINTb and MABROa (Table 3). Their average abundance was highest in rheocrenes $(38.1 \pm 14.8 \%)$ and lowest in rheohelocrenes $(26.6 \pm 19 \%)$, but also not significantly different between spring types (Table 4).

Considering only samples with adequate valve counts, IPS values showed little variation (average $16.0 \pm 0.9$ S.D.), ranging from 13.9 (MATERb) to 17.9 (KWINTb; Table 3). The average for Rott's trophic index was 2.5 \pm 0.3 . It was close to 2 for KRINDa, KRINDb, ENA$\mathrm{MEb}, \mathrm{MABROb}$, which would be considered mesotrophic-eutrophic and at least 2.5 (eutrophic) for all other samples. MATERb obtained the highest score (2.9, euto polytrophic) due to the abundance of Planothidium frequentissimum and Gomphonema parvulum. All spring types were very similar for both indices (Table 4). Only MATERb presented a substantial abundance of taxa considered to indicate anthropogenic alteration in Hessen $(20.3 \%)$, resulting in a somewhat higher average for rheohelocrenes. Overall, the diatom results suggested the best water quality for KRIND, whereas considerable impairment was deemed likely for MATERb. The latter site was close to a road but chemically stood out only by a slightly higher iron content; SRP nor nitrogen levels were in any way exceptional.

Correlations between assemblage-derived metrics remained modest throughout (Table 5). In agre-
Table 4. Characteristics of springs with Cratoneurion in Flanders grouped by geomorphological type (average with standard deviation; none of the metrics differed significantly between spring types at $\mathrm{p} \leq$ 0.05). See Table 3 and text.

\begin{tabular}{lccc}
\hline metric & hygropetric & rheocrene & $\begin{array}{c}\text { rheohelo- } \\
\text { crene }\end{array}$ \\
\hline taxa & $57.3 \pm 8.8$ & $67.1 \pm 14.8$ & $63.0 \pm 23.2$ \\
\# R & $4.5 \pm 1.3$ & $8.1 \pm 2.6$ & $6.4 \pm 2.5$ \\
$\% \mathrm{R}$ & $2.1 \pm 1.4$ & $5.0 \pm 3.7$ & $6.5 \pm 5.5$ \\
\# E & $4.5 \pm 0.6$ & $6.9 \pm 3.2$ & $7.6 \pm 2.5$ \\
$\% \mathrm{E}$ & $0.9 \pm 0.2$ & $3.7 \pm 2.7$ & $4.8 \pm 1.6$ \\
$\% \mathrm{X}$ & $31.9 \pm 18.2$ & $38.1 \pm 14.8$ & $26.6 \pm 19.0$ \\
$\mathrm{IPS}$ & $16.5 \pm 1.0$ & $15.8 \pm 0.5$ & $15.9 \pm 1.3$ \\
$\mathrm{TI}$ & $2.5 \pm 0.3$ & $2.6 \pm 0.2$ & $2.5 \pm 0.4$ \\
\% $\mathrm{A}$ & $0.8 \pm 0.6$ & $1.4 \pm 1.1$ & $5.1 \pm 10.1$ \\
\hline
\end{tabular}

Table 5. Significant Pearson or Spearman correlations between assemblage metrics and environmental variables $(\mathrm{p} \leq 0.05, \mathrm{p} \leq 0.01$, $\mathrm{p} \leq 0.001)$.

\begin{tabular}{|c|c|c|c|c|c|c|}
\hline & $\# \mathbf{R}$ & $\% \mathbf{R}$ & $\# \mathbf{E}$ & $\% \mathbf{E}$ & IPS & $\% \mathrm{~A}$ \\
\hline $\operatorname{taxa}$ & 0.57 & - & 0.70 & - & - & 0.48 \\
\hline$\% \mathrm{R}$ & - & 1 & - & - & - & - \\
\hline \# E & 0.53 & - & 1 & - & - & - \\
\hline$\% \mathrm{E}$ & - & - & 0.78 & 1 & - & - \\
\hline $\mathrm{TI}_{\text {DIA }}$ & 0.66 & - & - & - & -0.64 & - \\
\hline$\% \mathrm{~A}$ & - & - & - & - & -0.59 & 1 \\
\hline $\begin{array}{l}\text { bryophyte } \\
\text { cover }\end{array}$ & - & - & - & - & - & 0.56 \\
\hline tufa & - & - & - & -0.60 & - & - \\
\hline alkalinity & - & - & - & - & - & -0.52 \\
\hline $\mathrm{Ca}^{2+}$ & - & 0.55 & - & - & - & -0.48 \\
\hline
\end{tabular}

ement with expectations, IPS correlated negatively to $\mathrm{TI}_{\mathrm{DIA}}$ and the abundance of indicators for anthropogenic influence, but none of these indices related to obvious chemical clues for eutrophication. Notably, \% A related positively to bryophyte cover, but decreased somewhat with higher alkalinity and calcium. Abundance of rare taxa, however, increased somewhat with calcium. There was also a weak positive relation between $\%$ $\mathrm{A}$ and taxa richness. The number of RL taxa increased with taxa richness, the number of endangered taxa, as well as the trophic score. The number of taxa in both RL groups correlated only marginally, but the number of endangered taxa followed their abundance rather closely. Yet, only \% E declined significantly with more 
tufa limestone. Although none of the well-illuminated sites showed more than $25 \%$ xerotolerant diatoms, their abundance was not correlated to vegetation cover or shade.

Again excluding FONTEb and WIJNGa, Amphora pediculus $(16.9 \pm 14.6 \%)$, Planothidium lanceolatum $(9.2 \pm 10.6 \%)$, Achnanthidium jackii $(6.7 \pm 6.8 \%$ ) and $P$. frequentissimum $(5.8 \pm 6.0 \%)$ were the only species with an average abundance of at least $5 \%$. Besides these, Caloneis lancettula (Figure 3 e), Cocconeis euglypta, Humidophila brekkaensis, H. perpusilla, Encyonopsis microcephala, Eolimna minima, Gomphonema parvulum, Halamphora normanii, Hippodonta neglecta (Figure 3 t), Navicula tripunctata, Nitzschia amphibia, N. sinuata, Pinnularia kuetzingii (Figure 4 g-i), Rhopalodia operculata (Figure 4 z), Stauroneis thermicola, Staurosira venter and Staurosirella pinnata reached at least $10 \%$ in one or more samples. Overall, assemblages were dominated by smaller raphe-bearing taxa. Non-motile Pseudostaurosira, Staurosirella and Staurosira were only more prominent in KRINDb and, less so, KWINTa. Centrics were hardly present.

Most samples clustered in three groups (Table 4). Goup I was characterized mainly by Planothidium spp., Navicula veneta and Stauroneis separanda (Figure 5j), group II by Achnanthidium jackii, Amphora pediculus, Gomphonema angustatum and group III by Humidophila irata, Luticola acidoclinata, Pinnularia obscura morphotype2, Pseudostaurosira brevistriata and Stauroneis borrichii (Fig. 5a). Group III mainly comprised samples representing the Vaals aquifer, but clusters were not consistent with groundwater type, nor spring type or even site. Three samples were set apart, including both with skewed relative abundances due to low valve counts, as well as MOENb.

Indval analysis suggested a significant association of Diploneis krammeri (Indval $60 \%, \mathrm{p}=0.04$ ), D. separanda (Indval 73\%, p =0.03), Gomphonema micropus (Indval 63\%, p =0.04; Figure 3n), G. utae (Indval 82\%, p =0.02), Nitzschia alpina (Indval 81\%, $\mathrm{p}=0.01$; Figure 4b), N. pusilla (Indval 69\%, p =0.04), Stauroneis pseudagrestis (Indval 67\%, p =0.05; Figure 5i), S. separanda (Indval $69 \%, \mathrm{p}=0.03$ ) and $S$. smithii (Indval $89 \%, \mathrm{p}=0.03$ ) with the aquifer Tielt 3 , but this was represented by only 2 samples from the same spring. The listing of Gomphonema angustum as the only indicative species (Indval $76 \%, p=0.02$ ) for the Vaals aquifer (6 samples) could be somewhat more robust. The aquifers Heers (HORNEa), Borgloon (WIJNGa) and Moen (MOENb) were represented by single samples. The first mainly had Planothidium spp., small Stauroneis and Navicula veneta, the second mostly Frustulia amosseana, Stauroneis thermicola, Gyrosigma obtusatum and Humidophila brekkaensis. MOENb differed distinctly by a prevalence of Encyonopsis microcephala, Nitzschia amphibia, N. sinuata, Pinnularia kuetzingii and Rhopalodia operculata.

Six taxa were indicative of hygropetric rheo- crenes: Cocconeis euglypta (Indval $68 \%, \mathrm{p}=0.008$ ), Navicula upsaliensis (Indval $64 \%, \mathrm{p}=0.02$; Figure 3 aa), Caloneis lancettula Indval 75\%, p =0.03), Hippodonta neglecta (Indval 50\%, p =0.03), Sellaphora pupula (Indval 50\%, p =0.03) and Amphora pediculus (Indval 57\%, p =0.04), two of rheocrenes: Stauroneis thermicola (Indval 76\%, $\mathrm{p}=0.03$ ) and $A$. copulata (Indval 55\%, p =0.05). Very few taxa indicated the longitudinal position of a sample within the drainage system: Caloneis leptosoma (mostly atypical linear forms with blunt apices; Figure 3f) and Humidophila brekkaensis occurred more where the water emerged (position A; Indval 66 and 69\% respectively, p = 0.04), Eolimna minima was found mostly within the zone of carbonate precipitation (C sites; Indval 85\%, $\mathrm{p}=0.02$ ). Amphora pediculus (Indval 57\%, p = 0.03), Cocconeis euglypta (Indval 63\%, p =0.02) and Sellaphora joubaudii (Indval 55\%, $\mathrm{p}=0.04$; Figure $4 \mathrm{ac}$ ) were more typical of sites with the highest amounts of calcite (categories 4-5), whereas Diploneis separanda (Indval $55 \%, \mathrm{p}=0.03$ ), Gyrosigma obtusatum (Indval 50\%, p $=0.03$ ), Hantzschia amphioxys (Indval 71\%, $\mathrm{p}=0.02$ ), Muelleria terrestris (Indval 50\%, p =0.04), Nitzschia pusilla (Indval 46\%, p=0.04), Pinnularia isselana (Indval $72 \%, \mathrm{p}=0.03$ ) as well as $P$. perirrorata (Indval $51 \%, \mathrm{p}=0.05)$ occurred somewhat more where encrustation was very limited (categories 1 and 2).

DCA indicated limited species turnover (axis 12.9 S.D., axis 23.2 S.D.) and species-environment relations were explained best by RDA. Because its potassium concentration was as least four times higher (25.5 mg. $\left.\mathrm{l}^{-1}\right)$ than in all other springs and levels of sulphate, magnesium and sodium were also the highest measured, the sample MOENb behaved as an extreme outlier in RDA. Consequently it was neglected in further analyses. Sample FONTEb was included passively because its very poor diatom content did not allow reliable abundance estimates, and samples MABROb and WIJNGa were down-weighted. Forward selection suggested that $\mathrm{pH}$, nitrate and magnesium explained species composition best, accounting jointly for $32.9 \%$ of the variation (Table 6). The marginal contributions of nitrate and magnesium were similar and slightly smaller than for $\mathrm{pH}$, with the response to nitrate depending more strongly on the condition of both additional variables (cf. $\Lambda_{1 \mathrm{c}} / \Lambda_{1}$; Table 7 ).

High $\mathrm{pH}$ was associated with more intense carbonate precipitation and higher abundance of Amphora pediculus, Caloneis lancettula and Navicula upsaliensis. With positive scores on the principal axis, magnesium aligned well with major anions, potassium, calcium and alkalinity. Gomphonema parvulum and Planothidium spp. were most abundant in more mineral-rich conditions. Also correlating positively with the first axis were phosphate and iron (the latter solely due to MATERb). Phosphorus will precipitate with calcium and iron as oxygenation increases and most of the samples away from the spring head indeed plotted on the left- 


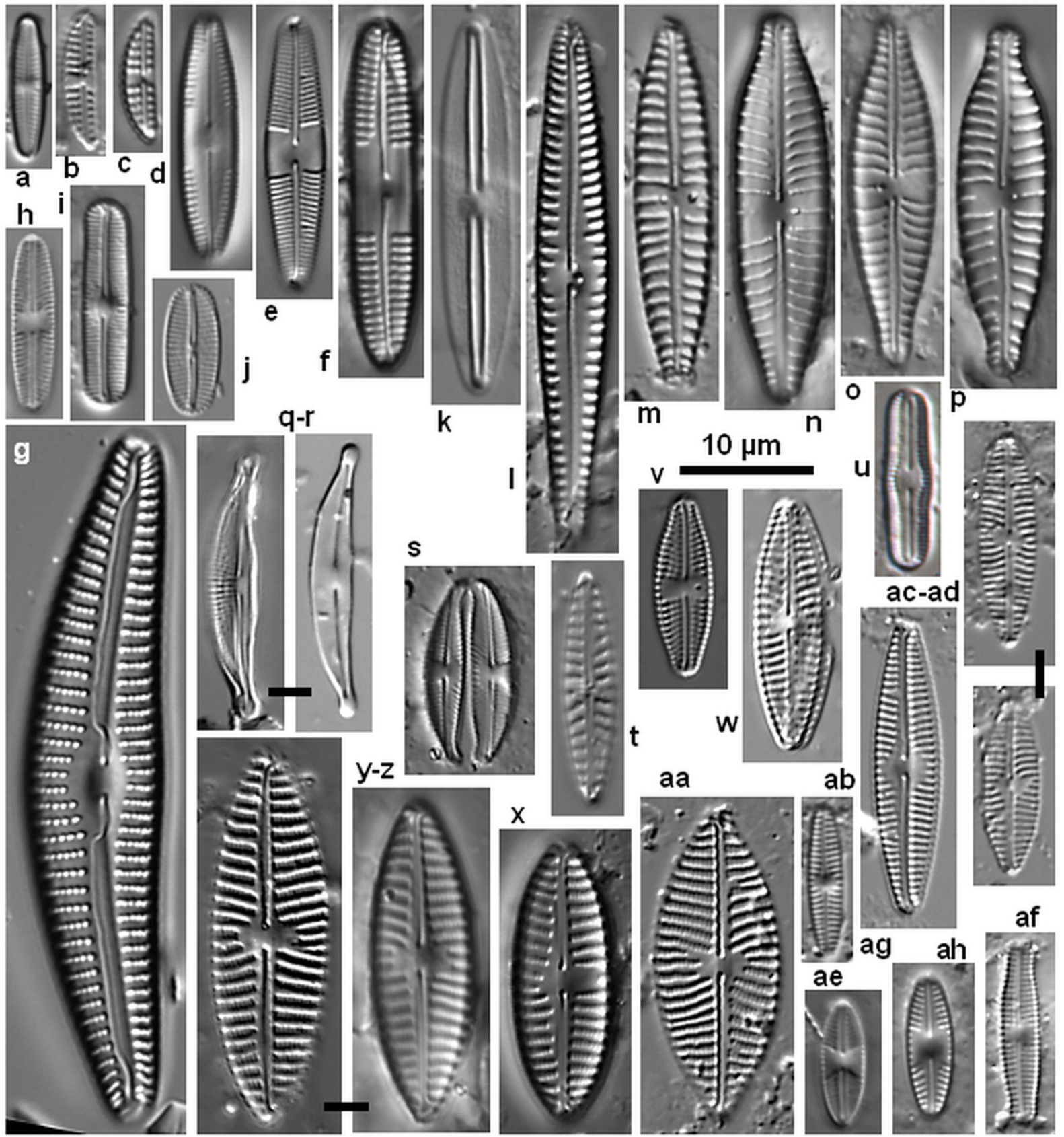

Fig. 3. (a) Achnanthidium jackii; (b) Amphora indistincta, (c) A. pediculus; (d) Caloneis fontinalis; (e) C. lancettula; (f) C. leptosoma (lack of triundulate margins and acute apices agree with C. leptosoma f. grunowii CLEVE-EuLER, which may need taxonomic reconsideration); (g) Cymbella alpestris; (h) Eolimna tantula; (i) Fallacia langebertaloti; (j) F. sublucidula; (k) Frustulia sp. BOTERa; (1) Gomphonema cf. designatum; (m) G. longilineare; (n) G. micropus; (o) G. micropus morphotype "aequalidictum"; (p) G. micropus var. aequale (the distinctly curved raphe suggests it might be more appropriate to retain Gregory's species rank ); (q-r) Halamphora oligotraphenta (r. craticular valve); (s) H. sp. GALMAa; (t) Hippodonta neglecta; (u) Humidophila brekkaensis; (v) Luticola paramutica; (w) Navicula arctotenelloides; (x) N. aff. catalanogermanica; (y-z) N. moenofranconica; (aa) N. upsaliensis; (ab) N. aff. veneta; (ac-ad) N. sp. GALMAb; (ae) Navicula s.l. harderi; (af) N. s.l. ENAMEa; (ag) N. s.l. (Chamaepinnularia) obsoleta; (ah) N. s.l. sp. subseminulum. 


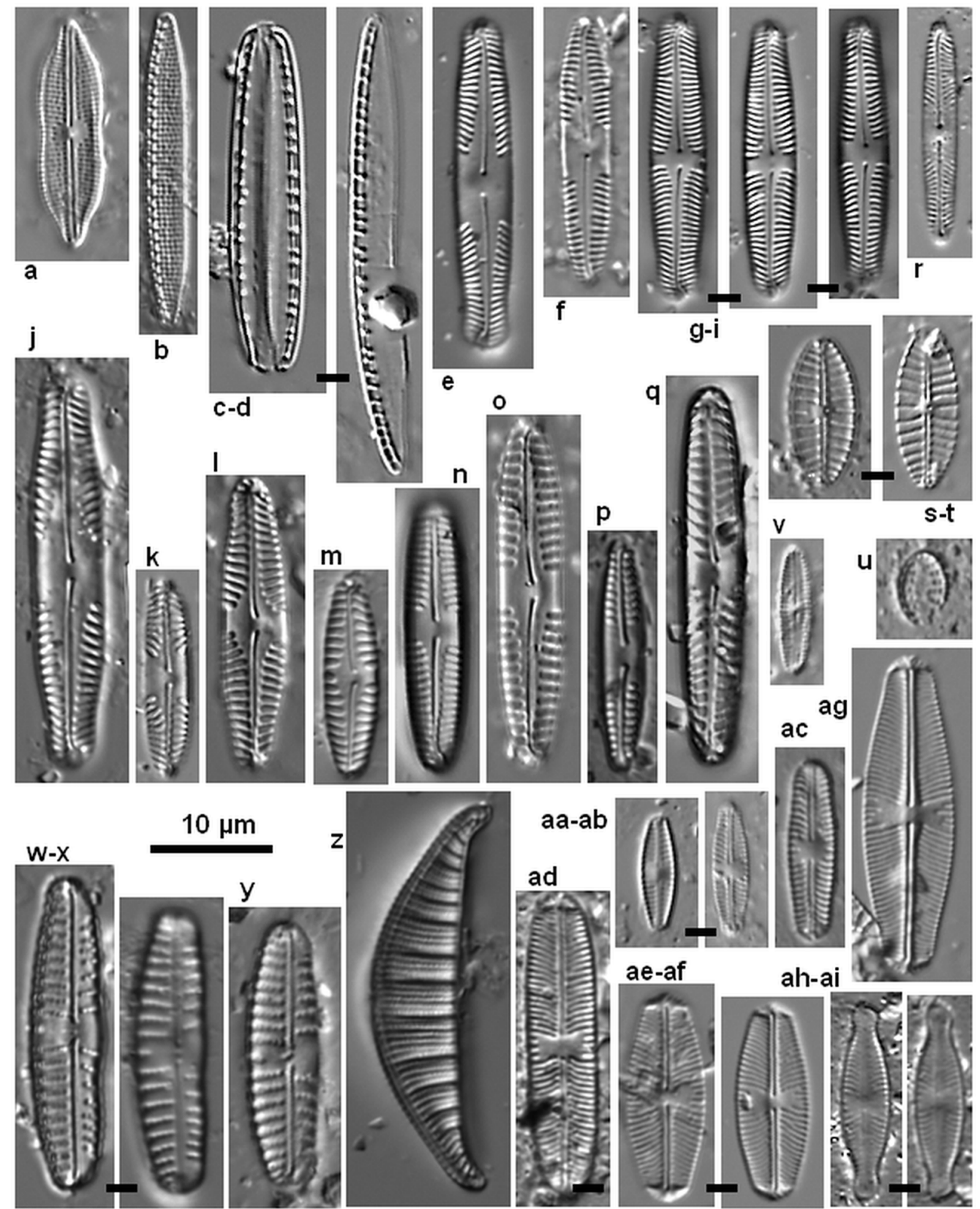

Fig. 4. (a) Neidiomorpha binodiformis; (b) Nitzschia alpina; (c-d) N. harderi; (e) Pinnularia bertrandii var. orleansiana; (f) P. frauenbergiana var. caloneiopsis; (g-i) P. kuetzingii; (j) P. marchica; (k) P. osoresanensis; (1) P. pisciculus; (m) P. sp. BOTERa1 (distinct from P. obscura); (n) $P$. sp. BOTERa2; (o) $P$. sp. FEELBa; (p) $P$. sp. FEELBc; (q) $P$. sp. GALMAa (close to $P$. palatina LANGe-Bert. \& Krüger); (r) $P$. sp. HORNEa (close to P. acoricola Hust. var. linearis WydrzycKa, LAnge-Bert. \& Metzeltin); (s-t) Placoneis sp. KRINDb (note presence of a stigma); (u) Pseudostaurosira sp. HORNEa; (v) Pulchella minutissima; (w-x) Reimeria uniseriata; (y) R. aff. uniseriata (note double rows of areolae on dorsal side); (z) Rhopalodia operculata; (aa-ab) Sellaphora cf. hafnerae; (ac) S. joubaudii; (ad) S. pseudopupula; (ae-af) S. pupula phenodeme little; (ag) S. pupula phenodeme tidy; (ah-ai) S. aff. sardiniensis. 
hand side. High nitrate samples tended to be from more shaded sites with lower mineral concentrations and had more Humidophila contenta, Gomphonema angustum, small fragilarioids (Staurosira, Staurosirella), as well as a good representation of Amphora pediculus. Away from this main gradient, a number of xerotolerant taxa (Luticola spp., Pinnularia obscura, Stauroneis muriella, Tryblionella debilis) clustered near the samples KWINTa and MABROa with high scores along the second ordination axis; opposite, Achnanthidium species obtained negative scores. This second axis appeared to integrate mineral content (magnesium associated with potassium and sodium as chlorides and sulphates) and, to a lesser extent, plant cover (negatively) versus shade (positively).

In accordance with their inferred water quality, RDA positioned KRIND and MATERb oppositely, whilst $\mathrm{TI}_{\mathrm{DIA}}$ scores for their closest satellites, MABROb and KWINTb and BOTERa, respectively, agreed well with a similar differentiation. Not all differences in $\mathrm{TI}_{\text {DIA }}$ concurred well with a similar ordination position, however, as evident from ENAMEb and KWINTb which shared the same value (2.3) and the samples from FEELB and GALMA with higher $\mathrm{TI}_{\mathrm{DIA}}(2.5-2.8)$.

\section{Discussion}

With 50 taxa/sample on average, excluding observations outside counts, and local $(\alpha)$ diversity exceeding 60 otherwise, local species richness was high compared to that of spring epilithon in the Italian Alps (average number 14, counts of 450 valves; SPITALE et al. 2012), Poland (18 with 300 valves counted; WoJTAL \& Soвсzyк 2012), the German Spessart Muschelkalk and Wetterau marl-loess regions (22 and about 30 in 400 valves, respectively; Werum \& LANGE-BERTALOT 2004), as well as central France (18; BERTRAND et al. 1999). In spite of intensive searching, Cantonati \& LANGE-Bertalot (2010) observed only 12 to 37 taxa on various substrates in SE Germany. Sabater \& Roca (1992; see Gieserich \& Kofler 2010 for studies with supporting observations), suggested that shear stress constrained species richness in springs and the lack of extreme flows probably contributed to the observed higher $\alpha$ diversity. Comparable flow conditions may also have resulted in similar taxon richness for all geomorphological types. On the other hand, ABOAL et al. (1998) reported that diversity increased with altitude. Species richness in springs is often considered to be lowered by impairment, although it is not always clear whether this is due to reduced habitat heterogeneity from morphological alteration (ТАХBÖCK \& PrEISIG 2007), altered water quality (BERTRAND et al. 1999), or both. No relation to IPS, TI ${ }_{\text {DIA }}$ or physical-chemical indications of eutrophication was observed in the present study. Rather, $\alpha$ diversity tended to increase along with the abundance of taxa associated with impact in comparable German springs. This shows that higher species richness is not an unequivocal sign of more natural or oligotrophic conditions and should be considered strictly within its proper geographical context.

Although physical-chemical conditions were largely similar among sites, regional taxonomic richness $(\gamma$ diversity) of calcareous spring habitats in Flanders also appeared to be high compared to other regions. SABATER \& Roca (1992), for example, noted slightly less than 200 taxa in 28 presumably pristine Pyrenean springs on limestone, granite and slates. Seventeen Swiss springs, also presenting a wider conduc-

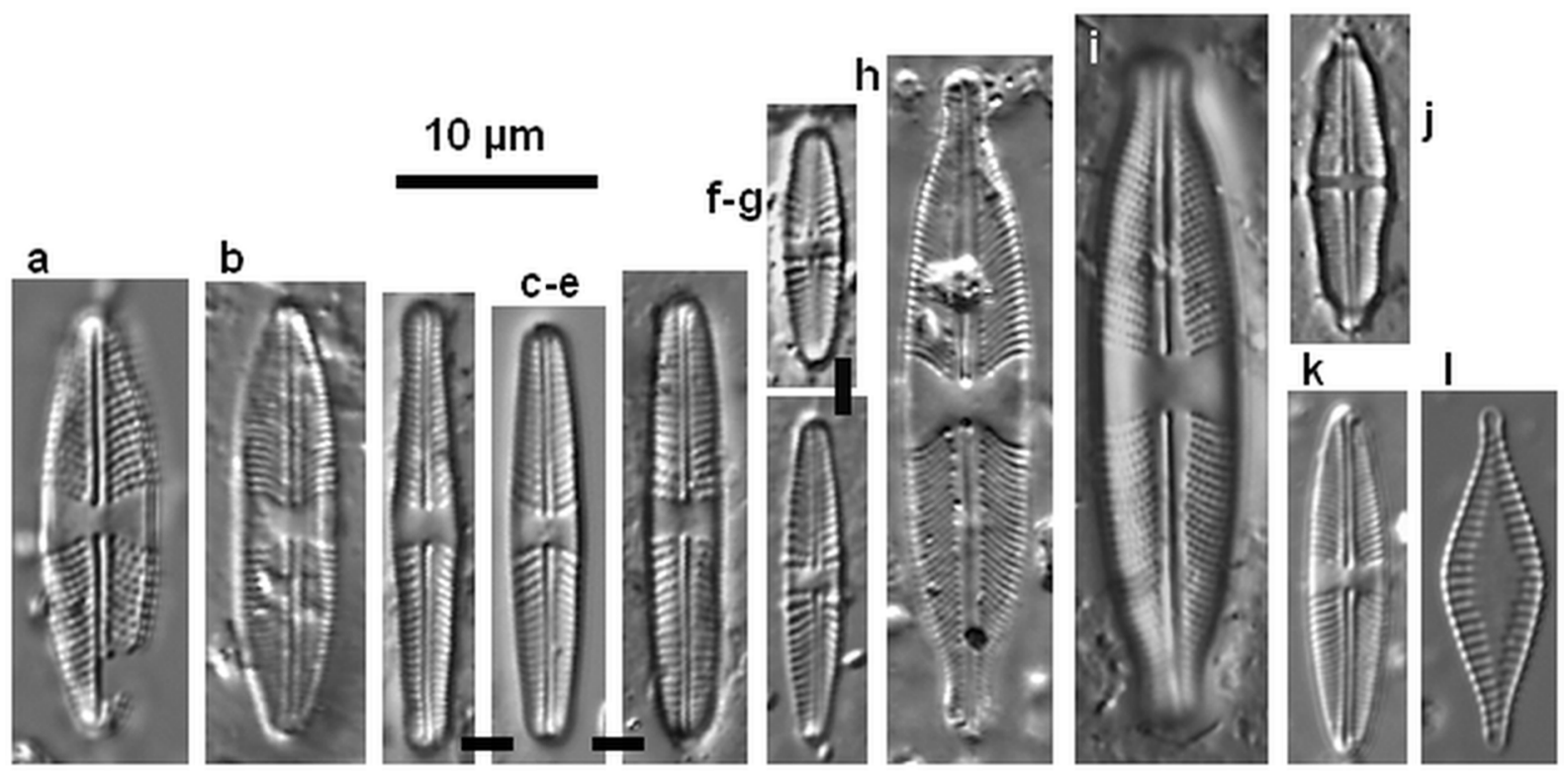

Fig. 5. (a) Stauroneis borrichii; (b) S. lundii; (c-e) S. muriella; (f-g) S. parathermicola; (h) S. pseudagrestis; (i) S. schimanskii; (j) S. separanda; (k) S. sp. FEELBa; (1) Synedrella parasitica. 


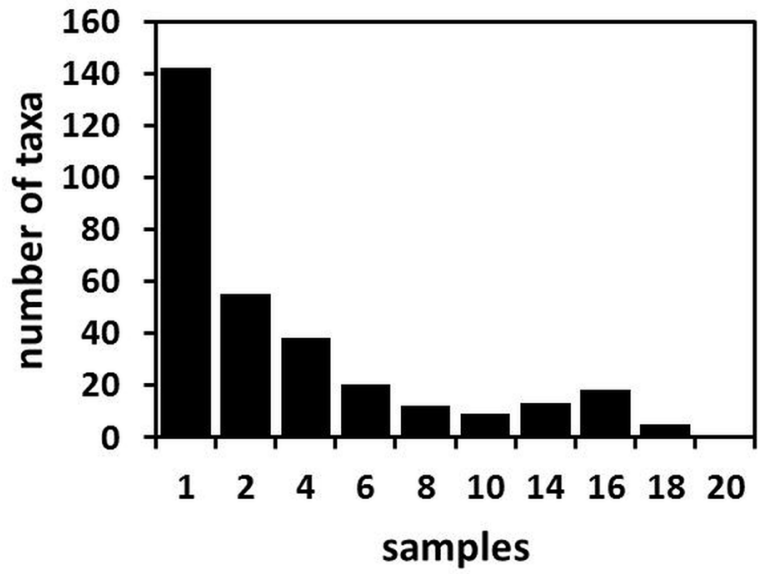

Fig. 6. Frequency distribution of taxa.

tivity range and no obvious signs of pollution, yielded only 136 taxa (ТАхвӧск \& Preisig 2007) and 197 taxa were noted in 52 samples from 27 Austrian springs and spring streams (GESIERICH \& Kofler 2010). Thirteen springs in the Western Carpathians yielded 188 taxa, with 114 occurring in calcareous fens (FrÁNKOVÁ et al. 2009). Werum \& Lange-Bertalot (2004) examined 97 springs from regions with varying geology in SWGermany (Hessen) but recorded only one hundred taxa more than the $300+$ tallied here. Although comparisons of species numbers are always hampered by differences in methodology and adherence to counts of a few hundred valves leads to severe truncation of diatom inventories to more abundant taxa (see also GERECKE et al. 2011), the differences in richness seem genuine, taxonomic resolution also offering only a partial explanation. Strong heterogeneity of spring assemblages is well established (Bertrand et al. 1999; CANTONATI et al. 2012b) and the very low frequency of a large majority of all taxa leaves little doubt that $\gamma$ diversity of carbonate-precipitating springs in Flanders is far from accounted completely. The present observations were restricted to a single substrate type and occasion and therefore neglected much of the heterogeneity in local habitat conditions. Epipelic and bryophytic assemblages are likely to differ in composition (e.g. FránKOvÁ et al. 2009 ). In northern Italy, CANTONATI et al. (2012b) found the highest numbers of diatom taxa on bryophytes, a substrate offering diverse microhabitats as well as pockets with accumulative conditions and little scouring, and significant substrate preference for a considerable number of taxa. Other studies, however, found that species richness on mosses was quite similar to that of epilithon (e.g. WoJTAL \& SobCzyK 2012), or even lower (Hustedt 1945). We observed no relation between bryophyte abundance and species richness, but expect that their examination will yield additional taxa. Seasonal variation is also not without possible consequence for assemblage composition (e.g. CANTONATI 2001, BATTEGAZZORE et al. 2004) but was not yet addressed, either. In addition, Cratoneurion springs representing the aquifers Lede, Tielt 4 and Hannuit await investigation. Some of these show a more distinct chemical composition, e.g. water emanating from the formation of Lede presents higher chloride levels than observed in any of the springs examined here.

Two samples probably offered a very incomplete picture of local diversity. The very low valve concentrations in these samples may have various reasons and it is not clear which contributed exactly. CANTONATI $\&$ Spitale (2009) and Cantonati et al. (2012b) made similar observations in a few iron-rich springs, but this was not an apparent feature here. FráNKOvÁ et al. (2009) observed poor valve silicification in petrifying springs, concurring with somewhat lower silica concentrations than in less mineral-rich fens. The solubility of biogenic silica increases markedly at $\mathrm{pH}>8.5$ and such values may develop in carbonate-precipitating biofilms (BISSET 2008). Also, primary productivity of springs can be relatively low in comparison to other lotic habitats due to low light and nutrient availability or transient water supply. In such conditions, the phytobenthos may become depleted more easily by grazing or scouring. Moreover, extensive covering by leaf litter until shortly before sampling would limit diatom growth and, perhaps, even enhance microbial decomposition facilitating valve dissolution (EHRLICH et al. 2010).

In agreement with many alkaline springs elsewhere in Europe (e.g. Round 1957; REICHARDT 1994; FrÁnKová et al. 2009; Gesierich \& Kofler 2010; TAXBÖck \& Preisig 2007; Werum \& Lange-Bertalot 2004; WoJTAL \& Sobczyк 2012), the core assemblage consisted of a series of more or less ubiquist taxa (Achnanthidium jackii, A. minutissimum, Amphora pediculus, Cocconeis placentula group, Planothidium frequentissimum, P. lanceolatum). Relative to other European spring areas, Stauroneis, but also Caloneis and Gomphonema appeared to be particularly abundant. In spite of the more alkaline conditions, Pinnularia species were not uncommon, either. In particular, small-celled taxa of these genera occurring widely in subaerial environments were well represented, e.g. Caloneis fontinalis, Gomphonema micropus, Stauroneis thermicola, $S$. muriella, Pinnularia isselana and P. obscura. Several of the species considered typical for limestone formation by REICHARDT (1994) were frequent companions (Caloneis fontinalis, Fallacia langebertalotii, Gomphonema angustum), but Caloneis alpestris, Diploneis minuta and Cymbopleura subaequalis remained rare. Of the taxa occurring more often in the marl-loess than in the Musschelkalk parts of Hessen according to Werum \& Lange-Bertalot (2004), Caloneis bacillum sensu auct. nonnull., Diploneis spp., Navicula veneta, Nitzschia dissipata and Stauroneis smithii were encountered regularly, but so were Achnanthidium jackii, Amphora copulata, Fallacia langebertalotii and Navicula s.l. harderi for which the reverse was observed. Overall Cymbella, Denticula, Encyonema, 


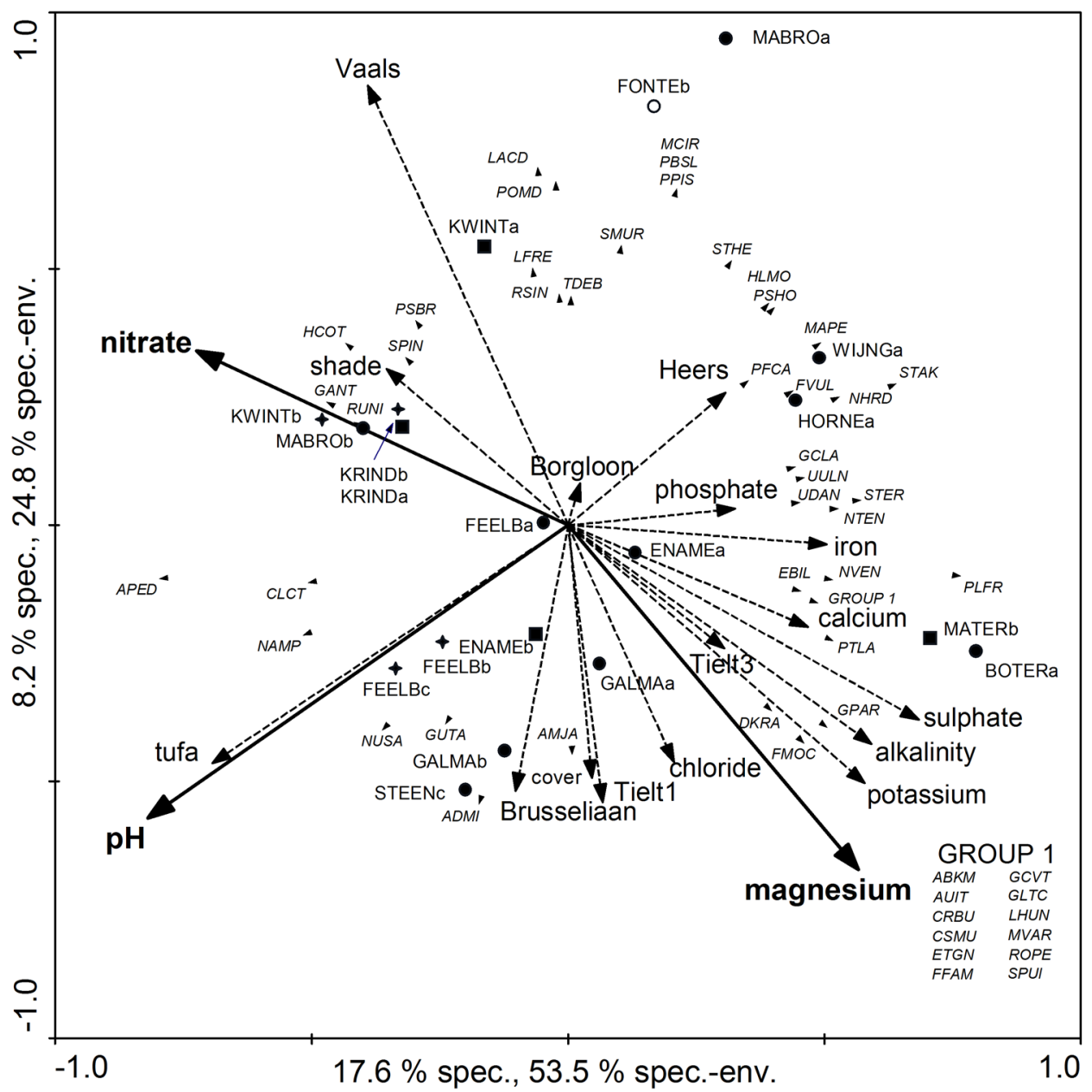

Fig. 7. Ordination plot of samples ( hygropetric rheocrene, $\mathbf{C}$ rheocrene, $\boldsymbol{\square}$ rheohelocrene), taxa (arrow heads), active variables (full-line arrows) and selected passive variables (dashed line arrows) for the two principal RDA axes. Only taxa with fit $\geq 20 \%$ are shown. (AMJA) Achnanthidium jackii; (ADMI) A. minutissimum; (ABKM) Adlafia brockmanni; (APED) Amphora pediculus; (AUIT) Aulacoseira italica; (CRBU) Craticula buderi; (CLCT) Caloneis lancettula; (CSMU) Chamaepinnularia submuscicola; (DKRA) Diploneis krammeri; (ETGN) Epithemia turgida var. granulata; (EBIL) Eunotia bilunaris; (FMOC) Fallacia monoculata; (FFAM) Fragilaria famelica; (FVUL) Frustulia vulgaris; (GANT) Gomphonema angustum; (GCLA) G. clavatum; (GCVT) G. clavatulum; (GLTC) G. laticollum; (GPAR) G. parvulum; (GUTA) G. utae; (HCOT) Humidophila contenta; (HLMO) Halamphora montana; (LHUN) Lemnicola hungarica; (LACD) Luticola acidoclinata; (LFRE) L. frequentissima; (MAPE) Mayamaea atomus var. permitis; (MVAR) Melosira varians; (MCIR) Meridion circulare; (NHRD) Navicula s.l. harderi; (NTEN) Navicula tenelloides; (NUSA) N. upsaliensis; (NVEN) N. veneta; (NAMP) Nitzschia amphibia; (PBSL) Pinnularia borealis var. sublinearis; (PFCA) P. frauenbergiana var. caloneiopsis; (POMD) P. obscura morphotype 2; (PPIS) P. pisciculus; (PSHO) P. schoenfelderi; (PSBR) Pseudostaurosira brevistriata; (PLFR) Planothidium frequentissimum; (PTLA) P. lanceolatum; (RSIN) Reimeria sinuata; (RUNI) R. uniseriata; (ROPE) Rhopalodia operculata; (SPUI) Sellaphora pupula phenodeme little; (SMUR) Stauroneis muriella; (STAK) S. tackei; (STHE) S. thermicola; (SPIN) Staurosirella pinnata; (STER) Surirella terricola; (TDEB) Tryblionella debilis; (UDAN) Ulnaria danica; (UULN) U. ulna.

Encyonopsis and Psammothidium were poorly represented. Brachysira, Diatomella, Rossithidium and Tetracyclus lacked entirely, as well as Achnanthidium pyrenaicum (Hust.) KoBAyAsi, Diatoma hiemale (RоTн) HeIB. and D. mesodon (EHr.) Kütz. These taxa often dominate in more elevated regions and high-flow conditions (e.g. Hustedt 1945; Sabater \& Roca 1992; Gesierich \& Kofler 2010). Psammothidium grischunum (Wuthrich) BukTh. \& Round and Planothidium dubium (GRUN.) Round \& BuKTH., both frequent on limestone in Germany (Werum \& LANGE-Bertalot 2004), also remained absent, similar to many taxa common in Cratoneurion habitat elsewhere (e.g. Caloneis tenuis (Greg.) Krammer, Delicata delicatula (Kütz.)
KrAmmer, Encyonopsis falaisensis (GRUN.) KRAMMER, Epithemia argus (EHr.) Kütz., Eucocconeis flexella (Kütz.) CL., E. laevis (Kütz.) MeISter, Eunotia arcubus NöRPEL-SCHEMP \& LANGE-BerT., Hannea arcus (Ehr.) Patrick; Ector \& Iserentant 1988; Iserentant 1988; FránKová et al. 2009; Cantonati \& Lange-BerTALOT 2010). Together with the better representation of fragilarioid taxa, this resulted in a somewhat stronger resemblance to assemblages found in limnocrenes (WoJTAL et al. 2009). Many of the above-mentioned species occur elsewhere in Flanders, e.g. in calcareous fens, rendering their apparent lack in these springs unexpected. Differences in thermal regime or trophic conditions could be involved, but it should be noted 
Table 6. Characteristics of RDA for diatom assemblages from calcareous springs in lower Belgium with $\mathrm{pH}$, nitrate and magnesium as active variables (MOENb excluded, FONTEb passive, MABROEb and WIJNGa down-weighted).

\begin{tabular}{llll}
\hline axis & $\mathbf{1}$ & $\mathbf{2}$ & $\mathbf{3}$ \\
\hline eigenvalue $(\Lambda)$ & 0.18 & 0.08 & 0.07 \\
species-environment correlation & 0.91 & 0.81 & 0.89 \\
$\%$ species relations explained & 17.6 & 25.8 & 32.9 \\
$\%$ species-environment explained & 53.5 & 78.3 & 100 \\
\hline
\end{tabular}

Table 7. Characteristics of constrained RDAs of diatom assemblages from calcareous springs in lower Belgium with $\mathrm{pH}$, nitrate or magnesium as active variables (MOENb excluded, FONTEb passive, MABROEb and WIJNGa down-weighted).

\begin{tabular}{|c|c|c|c|c|c|c|c|c|c|}
\hline & \multirow[b]{2}{*}{$\kappa_{1}(\%)$} & \multicolumn{3}{|c|}{ marginal effects } & \multicolumn{4}{|c|}{ conditional effects } & \multirow[b]{2}{*}{$\kappa_{1 \mathrm{c}} / \boldsymbol{K}_{1}$} \\
\hline & & $\mathbf{F}$ & $\mathbf{P}$ & $\boldsymbol{K}_{1} / \boldsymbol{K}_{2}$ & $\boldsymbol{K}_{1 \mathrm{c}}(\%)$ & $\mathbf{F}$ & p & $\hat{\Lambda}_{1 \mathrm{c}} / \boldsymbol{K}_{2 \mathrm{c}}$ & \\
\hline $\mathrm{pH}$ & 14.5 & 2.7 & 0.003 & 1.03 & 11.5 & 2.4 & 0.002 & 0.87 & 0.79 \\
\hline $\mathrm{NO}_{3}{ }^{-}-\mathrm{N}$ & 12.8 & 2.3 & 0.004 & 0.68 & 7.9 & 1.7 & 0.038 & 0.60 & 0.62 \\
\hline $\mathrm{Mg}^{2+}$ & 11.0 & 2.0 & 0.025 & 0.53 & 9.0 & 1.9 & 0.009 & 0.68 & 0.82 \\
\hline
\end{tabular}

that $\mathrm{TI}_{\mathrm{DIA}}$ values were similar to those on marl in Germany and largely within the range observed in northern Italy (ANGeLI et al. 2010). Overall, and in analogy of their moss vegetation (Oosterlynck \& De Bie in press), it appears that, the diatom flora of these springs presents a pauperized facies of the Cratoneurion elsewhere as far as 'boreo-alpine' taxa are concerned, somewhat dampening expectations regarding their possible role as least-impaired habitat relicts (CANTONATI et al. 2012a).

In line with the previous, the representation of taxa featuring on the Central European Red List was lower than reported for springs in many other regions (e.g. Cantonati 1998; TAXвÖck \& Preisig 2007; Angeli et al. 2010; Werum 2001; CAntonati \& Lange-BertALOT 2010; Gesierich \& Kofler 2010). Still, this was more a matter of relative abundance than taxa numbers, as more than 70 (c. $25 \%$ ) of all taxa might be considered rare or endangered, making up about $1 / 5$ of all taxa in a sample, but only $1 / 12$ of all individuals. Absence of base-line data on springs in the region so far also prevents to conclude that their relative scarcity reflects a more impacted condition. Notably, the historical 'background' representation of such taxa in standing waters within the same region is quite low (DENys 2000). The Red List predominantly focusses on oligotraphentic taxa occurring either in mineral-poor waters, or where phosphorus availability is much reduced by formation of non-soluble precipitates, such as carbonates. The percentage abundance of rare taxa indeed increased somewhat with calcium content. The conservation value of hygropetric helocrenes was not exceptional with regard to their diatom composition.
Neither was this the case for sites where more limestone occurred, which even showed a lower abundance of endangered taxa. This suggests that, although an essential criterion to delimit habitat type 7220 , the amount of already deposited carbonate may not be very informative to assess its local conservation status in this region. On the other hand, the calcium content of the emerging water regulates the nutrient status, buffering against eutrophication.

Achnanthidium minutissimum, Amphora pediculus, Caloneis lancettula, Cocconeis euglypta, Navicula upsaliensis, Nitzschia amphibia and Sellaphora joubaudii characterized more intensive calcite formation, whereas taxa with an upright stature (Eunotia bilunaris, Gomphonema clavatum, Ulnaria spp.), growing in mucilaginous colonies (Frustulia vulgaris, Gyrosigma obtusatum, Mayamaea atomus var. permitis), or adapted to semi-terrestrial conditions (Halamphora montana, Hantzschia amphioxys, Stauroneis thermicola) favoured the opposite. So far, it remains unclear to which extent subtle differences in water chemistry, flow or substrate characteristics contributed to this distinction. Hygropetric springs presented a somewhat higher number of characteristic taxa, which appears to align with their regional paucity (SPITALE et al. 2012).

In general, xerotolerant diatoms (e.g. Humidophila spp., Halamphora spp., Hantzschia spp., Luticola spp.) were common. Their abundance in springs may relate to periodic desiccation, e.g. due to water abstraction (Werum \& LANGe Bertalot 2004; CANTONATI et al. 2012b), but is also expected to be higher in rheohelocrenes where slope erosion and small-scale variation in wetness should be most pronounced, as 
well as in hygropetric rheocrenes where the water film is thin and often intermittent. Although, the representation of xerotolerant diatoms was actually the lowest in rheohelocrenes and highest in rheocrenes, within-type variation overruled possible differences. If not merely due to unrepresentative sampling, conditions may well have been too similar to result in a clear pattern. Likewise, a relation to vegetation cover could only be hinted at. However, more precise estimators of soil inputs may be required, as cover only presents a crude proxy.

Exploration of environmental relations was hampered in the present study by limited data and conclusions should be drawn cautiously. Clearly, the artificial site MOEN was the most prominent example of environmental species sorting. In spite of its recent nature, the assemblage comprised a range of 'typical spring diatoms', including several rare ones, illustrating that even sparsely occurring taxa will rapidly colonize suitable habitat. Although EC was not substantially different from several other springs, the main constituents of its assemblage were different from all other investigated springs and included several species that are considered to indicate higher conductivities (e.g. Nitzschia amphibia, N. sinuata, Pinnularia kuetzingii, Rhopalodia operculata). However, concentrations and ratios of specific ions were unique, presumably due to the mineralogical composition of the local aquifer of Tertiary marine deposits.

For the remaining sites, only $\mathrm{pH}$, nitrate and magnesium related to some extent with assemblage composition. These variables, or close correlates, were repeatedly shown to structure diatoms of springs and headwaters, substantiating that the detected pattern was not spurious. We did not include direct measures of current but physical effects were probably less influential than in torrential springs (SABATER \& ROCA 1990, 1992). Where $\mathrm{pH}$ was highest and limestone formation most prominent, assemblage composition was closer to that of Alpine rheocrenes on limestone with nitrate enrichment or shading, or similar rheocrenes with periodic dessication where abundance of Humidophila spp. was higher (CANTONATI et al. 2012b). Whereas Achanthidium jackii and A. minutissimum obtained similar ordination scores in the present analysis, WERUM \& LANGe-Bertalot (2004) found that these taxa differentiated springs of the Muschelkalk region presenting water with a higher mineral content $\left(\mathrm{EC}_{25}>700\right.$ $\mu \mathrm{S} . \mathrm{cm}^{-1}$ ) from those on loam-marl in Hessen. Such differences in the response of individual taxa once again illustrate the importance of geographic regionalization.

Although shade was found to be important elsewhere (CANTONATI 1998; CANTONATI \& PipP 2000; CANTONATI \& SPITALE 2009), it was not singled out as such in this case. Its alignment with nitrate prevented to establish a clear response, but it was also fairly intense at most places. Nitrate sensitivity was demonstrated for the diatom phytobenthos in many spring systems (e.g. Cantonati 1998; Fránková et al. 2009; Angeli et al. 2010; HÁsKovÁ et al. 2011). Nitrate in spring water is considered to indicate anthropogenic impact but is modulated by the degree of retention and transformation in the soil and subsoil, as well as biological uptake before and after emergence (NIEDERMAYr \& SCHAGERL 2010). Of the common indicators for nitrate enrichment in other regions (CANTONATI et al. 2007, 2012b), only Amphora pediculus reacted more or less consequently. Together with other taxa tolerating organic pollution (Craticula buderi, Fallacia monoculata, Gomphonema parvulum, Lemnicola hungarica, $\mathrm{Na}$ vicula veneta, Planothidium frequentissimum), Planothidium lanceolatum showed a negative relationship to inorganic nitrogen but aligned with higher mineral content (viz. chloride, potassium and sulphate). This suggests that, although it might be possible to identify the predominant form of nitrogen and cause of pollution from its diatom signature, generalisations about the indicative meaning of individual taxa may be less straightforward.

Several issues remaining unresolved, the results of this preliminary survey and considerably more extensive studies carried out abroad point out that the microphytobenthos, with diatoms in particular (GERECKE et al. 2011), represents a major source of information on the functional and structural integrity, future prospects and regional peculiarities of petrifying springs. This may be especially welcome where their morphological development is more marginal and characteristic bryophyte communities less diverse, hampering a comprehensive elaboration of the favourable conservation status and environmental reference values of this endangered habitat type from more readily accessible macroscopic features.

\section{ACKNOWLedGements}

Sincere thanks to Steven De Saeger for help with field work, Arne Verstraeten and Piet De Becker for discussions and INBO laboratory staff for chemical analyses, as well as to Luc Ector and all others who provided publications. We also acknowledge the referees for all their suggestions.

\section{REFERENCES}

Aboal, M.; Puig, M.A. \& Prefas, M. (1998): Diatom assemblages in springs in Castellón province, Eastern Spain. - Algological Studies 90: 79-95.

Angeli, N.; Cantonati, M.; Spitale, D. \& Lange-Bertalot, H. (2010): A comparison between diatom assemblages in two groups of carbonate, low-altitude springs with different levels of anthropogenic disturbance. - Fottea 10: $115-128$.

Bertrand, J.; Renon, J.P. \& Monnier, O. (1999): Les diatomées des sources du rebord karstique de la Beauce de la region orléanaise. - Symbioses 1: 3-14

Battegazzore, M.; Morisi, A.; Gallino, B. \& Fenoglio, S. (2004): Environmental quality evaluation of Alpine springs in NW Italy using benthic diatoms. - Diatom 
Res. 19: 149-165.

Bissett, A.; De Beer, D. \& Schoon, R. (2008): Microbial mediation of stromatolite formation in karst-water creeks. Limnol. Oceanogr. 53: 1159-1168.

Cantonati, M. (1998): Diatom communities of springs in the Southern Alps. - Diatom Res. 13: 201-220.

CANTONati, M. (2001): The diatom communities of the liverwort Chiloscyphus polyanthus var. rivularis in a mountain spring-fed stream in the Adamello-Brenta Regional Park, Northern Italy. - In: JAHN, R.; KocioleK, J.P.; WITKowski, A. \& COMpère, P. (eds): Lange-Bertalot-Festschrift. - pp. 353-368, Gantner Verlag, Ruggell.

Cantonati, M.; Angeli, N.; Bertuzzi, E. \& Spitale, D. (2012b): Diatoms in springs of the Alps: spring types, environmental determinants, and substratum. - Freshwater Sci. 31: 499-524.

Cantonati, M.; Füreder, L.; Gerecke, R.; Jüttner, I. \& CoX, E.J. (2012a): Crenic habitats, hotspots for freshwater biodiversity conservation: toward an understanding of their ecology. - Freshwater Sci. 31: 463-480.

Cantonati, M. \& Lange-Bertalot, H. (2010): Diatom biodiversity of springs in the Berchtesgaden National Park. - Diatom Res. 25: 251-280.

Cantonati, M. \& Pipp, E. (2000): Longitudinal and seasonal differentiation of epilithic diatom communities in the uppermost sections of two mountain spring-fed systems. - Verh. Internat. Verein. Limnol. 27: 1591-1595.

Cantonati, M.; Rott, E.; Pfister, P. \& Bertuzzi, E. ( 2007): Benthic algae in springs: biodiversity and sampling methods. - In: Cantonati M., Pfister P. \& Bertuzzi E. (eds), The spring habitat: biota and sampling methods. - pp. 77-112, Museo Tridentino di Scienze Naturali, Trento.

Cantonati, M. \& Sitale, D. (2009): The role of environmental variables in structuring epiphytic and epilithic diatom assemblages in springs and streams of the Dolomiti Bellunesi National Park (south-eastern Alps). - Fund. Appl. Limnol. / Arch. Hydrobiol. 174/2: 117-133.

CEMAGREF (1982): Etude des méthodes biologiques d'appréciation quantitative de la qualité des eaux. - Q.E. Lyon-A.F. Bassin Rhône-Méditeranée-Corse, Lyon.

Delgado, C.; Ector, L.; Novais, M.H.; Blanco, S.; Hoffmann, L. \& PARDO, I. (2013): Epilithic diatoms of springs and spring-fed streams in Majorca Island (Spain) with the description of a new diatom species Cymbopleura margalefi sp. nov. - Fottea 13: 87-104.

DenYs, L. (1991): A check list of the diatoms in the Holocene deposits of the western Belgian coastal plain, with a survey of their apparent ecological requirements. I. Introduction, ecological code and complete list. - Professional Paper Belgische Geologische Dienst 246: 1-41.

Denys, L. (2000): Historical distribution of 'Red List diatoms' (Bacillariophyceae) in Flanders (Belgium). - Systematics and Geography of Plants 70: 409-420.

Dufrêne, M. \& Legendre, P. (1997): Species assemblages and indicator species: the need for a flexible asymmetrical approach. - Ecol. Monogr. 67: 345-366.

EPPINGER, R. \& THOMAs, P. (2007): Hydrogeologisch homogene zones ter bepaling van de nitraatkwetsbaarheid van het grondwater. - Water 28: 32-37.

European Commission (2007): Interpretation Manual of European Union Habitats. EUR 27. European Commission, DG Environment, Brussels.

Ector, L. \& Iserentant, R. (1988): Les diatomées des groupements fontinaux du Val de Bagnes (Valais, Suisse). Mémoires de la Société royale Botanique de Belgique 10: $12-16$.

Ehrlich, H.; Demadis, K.D.; Pokrovsky, O.S. \& Koutsoukos, P.G. (2010): Modern views on desilicification: biosilica and abiotic silica dissolution in natural and artificial en- vironments. - Chem. Rev. 110: 4656-4689.

Fránková, M.; Bojková, J.; PoulíčKovÁ, A. \& HáJeK, M. (2009): The structure and species richness of the diatom assemblages of the Western Carpathian spring fens along the gradient of mineral richness. - Fottea 9: 355-368.

Gerecke, R.; Cantonati, M.; Spitale, D.; Stur, E. \& WiedenBRUG, S. (2011): The challenges of long-term ecological research in springs in the northern and southern Alps: indicator groups, habitat diversity, and medium-term change. - J. Limnol. 70 (Suppl. 1): 168-187.

Gesierich, D. \& Kofler, W. (2010): Epilithic diatoms from rheocrene springs in the Eastern Alps (Vorarlberg, Austria). - Diatom Res. 25: 43-66.

Hájková, P.; Bojková, J.; FránKová, M.; Oprilová, V.; HájeK, M.; Kintrová, K. \& Horsá, M. (2011): Disentangling the effects of water chemistry and substratum on mossdwelling unicellular and multicellular micro-organisms in spring-fens. - J. Limnol. 70 (Suppl. 1): 54-64.

Hustedt, F. (1945): Diatomeen aus Seen und Quellgebieten der Balkan-Halbinsel. - Arch. Hydrobiol. 40: 867-973.

IsERENTANT, R. (1988): Les diatomées d'un travertine de pente ("cron") en Lorraine belge. - Mémoires de la Société royale Botanique de Belgique 10: 17-25.

Krammer, K. (1997a): Die cymbelloiden Diatomeen. Eine Monographie der weltweit bekannten Taxa. Teil 1. Allgemeines und Encyonema Part. - Bibl. Diatomol. 36: 1-382.

Krammer, K. (1997b): Die cymbelloiden Diatomeen. Eine Monographie der weltweit bekannten Taxa. Teil 1. Encyonema part., Encyonopsis and Cymbellopsis. - Bibl. Diatomol. 37: 1-469.

Krammer, K. (2000): Diatoms of Europe. Volume 1. The genus Pinnularia. - A.R.G. Gantner Verlag, Ruggell.

Krammer, K. (2002): Diatoms of Europe. Volume 3. Cymbella. - A.R.G. Verlag K.G., Ruggell.

Krammer, K. (2003): Diatoms of Europe. Volume 4. Cymbopleura, Delicata, Navicymbula, Gomphocymbellopsis, Afrocymbella. - A.R.G. Ganter Verlag K.G., Ruggell.

Krammer, K. \& Lange-Bertalot, H. (1999-2004): Bacillariophyceae. Teil 1-4. - In: Ettl, H.; Gerloff, J.; HeyNIG, H. \& Mollenhauer, D. (Hrsg.): Süsswasserflora von Mitteleuropa, Band 2. - 876 pp., 611 pp., 599 pp., $1-468,2^{\text {nd }}$ ed., Spektrum Akademischer Verlag, München - Heidelberg.

Lange-Bertalot, H. (2001): Diatoms of Europe. Volume 2. Navicula sensu stricto, 10 genera separated from Navicula sensu lato, Frustulia. - Gartner Verlag, Ruggel.

Lange-Bertalot, H.; Cavacini, P.; Tagliaventi, N. \& Alfinito, S. (2003): Diatoms of Sardinia. Rare and 76 new species in rock pools and other ephemeral waters. - Iconogr. Diatomol. 12: 1-438.

Lange-Bertalot, H. \& Steindorf, A. (1996): Rote Liste der limnischen Kieselalgen (Bacillariophyceae) Deutschlands. - Schriften-Reihe für Vegetationskunde 28: 633-677.

Levkov, Z. (2009): Diatoms of Europe. Volume 5. Amphora sensu lato. - 916 pp., Gartner Verlag, Ruggel.

Levkov, Z.; Metzeltin, D. \& Pavlov, A. (2013): Diatoms of Europe. Volume 7. Luticola and Luticopsis. - 697 pp., Koeltz, Königstein.

McCune, B. \& Mefford, M.J. (1999): PC-ORD. Multivariate Analysis of Ecological Data. Version 5.0. - MjM Software, Gleneden Beach.

Niedermayr, R. \& Schagerl, M. (2010): Structuring factors of the phytobenthos community along a mountain headwater (Kalkalpen National Park, Austria). - Fund. Appl. Limnol. 177: 93-104.

Oosterlynck, P. \& De BiE, E. (in press): Kalktufbronnen in Vlaanderen. Bryologische en abiotische karakterisering van een Natura 2000 habitattype op de rand van zijn verspreiding. - Rapporten van het Instituut voor Natuur-en 
Bosonderzoek, Brussel

Overloop, S.; Bossuyt, M.; Claeys, D.; D'hooghe, J.; Elsen, A.; EPPIngER, R. \& WUSTEnBerghs, H. (2011): Milieurapport Vlaanderen. Achtergronddocument 2011. Vermesting. Vlaamse Milieumaatschappij, www.milieurapport.be

Pentecost, A. (2005): Travertine. - Springer Verlag, Berlin Heidelberg.

PoulíčKová, A.; Bogdanová, K.; Hekera, P. \& Hájková, P. (2003): Epiphytic diatoms of the spring fens in the flysh area of the Western Carpathians. - Biologia 58: 749757.

ReICHARDT, E. (1994): Zur Diatomeenflora (Bacillariophyceae) tuffabscheidender Quellen und Bäche im Südlichen Frankenjura. - Berichte der Bayerischen Botanischen Gesellschaft 64: 119-133.

ReICHARDT, E. (1995): Die Kieselalgenflora (Bacillariophyceae) des Wachsenden Steins von Usterling. - Berichte der Bayerischen Botanischen Gesellschaft 65: 87-92.

ReICHARDT, E. (1999): Zur Revision der Gattung Gomphonema. Die Arten um G. affine/insigne, G. angustatum/micropus, G. acuminatum sowie gomphonemoide Diatomeen aus dem Oberoligozän in Böhmrn. - Iconogr. Diatomol. 8: 1-203.

Reichardt, E. (2004): Eine bemerkenswerte Diatomeenassoziation in einem Quellhabitat im Grazer Bergland, Österreich. Ein Beitrag zur Kenntnis seltener und wenig bekannter Diatomeen. - Iconogr. Diatomol. 13: 419479 .

Rott, E.; PipP, E.; Pfister, P.; van Dam, H.; Ortler, K.; Binder, N. \& PALL, K. (1999): Indikationslisten für Aufwuchsalgen In Österreichischen Fliessgewassern. Teil 2: Trophieindikation. - Bundesministerium für Land- und Forstwirtschaft, Vienna.

Round, F.E. (1957): A note on some diatom communities in calcareous springs and streams. - Journal of the Linnean Society Botany 55: 662-668.

SABATER, S. \& RocA, J.R. (1990): Some factors affecting distribution of diatom assemblages in Pyrenean springs. Freshwater Biol. 24: 493-507.

SABATER, S. \& RocA, J. R. (1992): Ecological and biogeographical aspects of diatom distribution in Pyrenean springs. - Br. Phycol. J. 27: 203-213.

Sanders, D.; Wertl, W. \& Rott E. (2010): Spring-associated limestones of the Eastern Alps: overview of facies, deposystems, minerals, and biota. - Facies: DOI 10.1007/ s10347-010-0252-y.

Spitale, D.; Leira, M.; Angeli, N. \& Cantonati, M. (2012): Environmental classification of springs of the Italian Alps and its consistency across multiple taxonomic groups. - Freshwater Sci. 31: 563-574.

Symoens, J.J. (1957): Les eaux douces de l'Ardenne et des regions voisines: les milieu et leur végétation algale. $-\mathrm{B}$ Soc. roy. Bot. Belg. 89: 111-314.

Symoens, J.J. (1960): Contribution à la flore algale de l'Ardenne et des regions voisines. - Bulletin du Jardin Botanique de l'Etat Bruxelles 30: 115-246.

TAXBÖCK, L. \& PreISIG, H.R. (2007): The diatom communities in Swiss springs: A first approach. - In: KusBer, W.-H. \& JAHN, R. (eds): Proceedings of the 1st Central European Diatom Meeting. - pp. 163-168, Botanic Garden and Botanical Museum Berlin-Dahlem, Berlin.

TER Braak, C.J.F. \& SMilauer, P. (2003): Canoco for Windows. Version 4.51. - Biometris, Wageningen.

Torrisi, M. \& Dell'Uomo, A. (2009): Diatomee bentoniche del corso superior di alcini fiumi centro-appenninici. - Studi Trentini di Scienze Naturali 84: 139-151.

van Dam, H.; Mertens, A. \& Sinkeldam, J. (1994): A coded checklist and ecological indicator values of freshwater diatoms from the Netherlands. - Netherlands Journal of
Aquatic Ecology 28: 117-133.

Werum, M. (2001): Diatomeen in Quellen hessischer Mittelgebirge: Gefährdung nach Roter Liste in Korrelation zu anthropogenen Eingriffen und Geologie. - In: JAHN, R.; Kociolek, J.P.; Witkowski, A. \& CompÈre, P. (eds): Lange-Bertalot-Festschrift. - pp. 369-381, Gantner, Ruggell.

Werum, M. \& Lange-Bertalot, H. (2004): Diatoms in springs from Central Europe and elsewhere under the influence of hydrogeology and anthropogenic impacts. - Iconogr. Diatomol. 13: 1-417.

WoJtal, A.Z. (2013): Species composition and distribution of diatoms assemblages in spring waters from various geological formations in southern Poland. - Biblioth. Diatomol. 59: 1-436.

Wojtal, A.Z. \& Soвczyк, Ł. (2012): The influence of substrates and physicochemical factors on the composition of diatom assemblages in karst springs and their applicability in water-quality assessment. - Hydrobiologia 695: $97-108$

WoJtal, A.Z.; WitKowski, A. \& Scharf, B. (2009): An approach to the recent environmental history of Pilica Piaski spring (southern Poland) using diatoms. - Hydrobiologia $631: 267-277$.

Supplementary material

the following supplementary material is available for this article:

Table S1. List of all observed taxa with their relative abundance and frequency, total valve count and attribution of samples to cluster groups. FONTEb and WIJNGa not considered in average and standard deviation. + outside count, * Red List group rare $(\mathrm{R}),{ }^{* *}$ Red List group endangered (E)

This material is available as part of the online article (http://fottea.czechphycology.cz/contents)

(C) Czech Phycological Society (2015)

Received January 8, 2015

Accepted February 24, 2015 